Allyl Sulfones as Precursors to Allylzincs in the Palladium-Catalyzed Zinc-ene Cyclization: Highly Efficient Synthesis of Enantiopure (-)-Erythrodiene

\author{
Kai Deng, Justin Chalker, Ao Yang and Theodore Cohen* \\ Department of Chemistry, University of Pittsburgh \\ Pittsburgh, Pennsylvania 15260
}

\title{
Index:
}

General Experimental Procedures, materials, and Instrumentation p. 1

$\begin{array}{ll}\text { Synthesis of Compounds 1-23 } & \text { p. } 2\end{array}$

$\begin{array}{ll}\text { Spectrum of Compounds 1-23 } & \text { p. } 16\end{array}$

General Experimental Procedures. All reactions were performed under an argon atmosphere in oven-dried $\left(110^{\circ} \mathrm{C}\right)$ flasks and standard precautions against moisture were taken. A dry ice/acetone bath was used to obtain a temperature of $-78{ }^{\circ} \mathrm{C}$. An ice bath was used to obtain $0{ }^{\circ} \mathrm{C}$. Silica gel 60 (40-60 $\mu \mathrm{m}$, Sorbent Technologies) was used for flash column chromatography. Thin-layer chromatography was performed on glass supported $250-\mu \mathrm{m}$ silica GF plates (Analtech). Visualization of TLC plates was accomplished with one or more of the following: $254 \mathrm{~nm}$ UV light; 7\% phosphomolybdic acid in ethanol; 5\% anisaldehyde in ethanol containing 5\% sulfuric acid and a trace amount of acetic acid; and/or aqueous $\mathrm{KMnO}_{4}(1 \%)$ with $\mathrm{NaOH}(.1 \%)$ and $\mathrm{K}_{2} \mathrm{CO}_{3}(6 \%)$. Anhydrous magnesium sulfate was used as the drying reagent. 
Materials. Commercial solvents and reagents were used as received with the following exceptions. Tetrahydrofuran (THF) and diethyl ether were distilled over sodium benzophenone ketyl. Methylene chloride and $\mathrm{CH}_{3} \mathrm{CN}$ were distilled over $\mathrm{CaH}_{2}$. Benzene was dried over melting sodium.

Instrumentation. Most ${ }^{1} \mathrm{H}$ and ${ }^{13} \mathrm{C}$ NMR spectra were recorded on Bruker DPX-300 spectrometer operating at $300 \mathrm{MHz}$ for ${ }^{1} \mathrm{H}$ and $75 \mathrm{MHz}$ for ${ }^{13} \mathrm{C}$ at $22{ }^{\circ} \mathrm{C}$ unless otherwise noted. Some ${ }^{1} \mathrm{H}$ and ${ }^{13} \mathrm{C}$ NMR spectra and two-dimensional NMR spectra were recorded on a Bruker AM-500 spectrometer. Chemical shift data are reported in units of $\delta(\mathrm{ppm})$ using $\mathrm{CHCl}_{3}$ as the internal standard: $\delta=7.27$ for ${ }^{1} \mathrm{H}$ NMR spectra and $\delta=77.09$ for ${ }^{13} \mathrm{C}$ NMR spectra unless indicated otherwise. Multiplicities are given as s (singlet), d (double), t (triplet), q (quartet), m (multiplet), and br (broad). Coupling constants, $J$, are reported in Hz. Infrared spectra were recorded on an IR/32 FT-IR spectrometer and are reported in wave numbers $\left(\mathrm{cm}^{-1}\right)$. Low and high-resolution mass spectra were recorded on a VG-70SE mass spectrometer in EI mode at $70 \mathrm{eV}$. Gas chromatographic mass spectra (GC-MS) analyses were performed on a Hewlett Packard 5890 Series II gas chromatograph equipped with a 5970 mass selective detector.

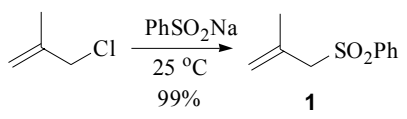

Methallyl Phenyl Sulfone (1) $)^{12}$ To a stirred solution containing $32.8 \mathrm{~g}(0.20 \mathrm{~mol})$ of benzenesulfinic acid sodium salt in $150 \mathrm{~mL}$ of DMF was slowly added $18.9 \mathrm{~g}(0.21$ mol) of 3-chloro-2-methylpropene at room temperature. After being stirred for $5 \mathrm{~h}$ at the same temperature, the reaction mixture was diluted with $500 \mathrm{~mL}$ of saturated 
aqueous $\mathrm{NH}_{4} \mathrm{Cl}$ and extracted with ethyl acetate $(200 \mathrm{~mL} \times 3)$. The combined organic layers were washed with water $\left(100 \mathrm{~mL}\right.$ x 3), and then brine, dried over $\mathrm{MgSO}_{4}$ and evaporated under reduced pressure. 2-methallyl phenyl sulfone (38.9 g, 99\%) was obtained without further purification. ${ }^{1} \mathrm{H} \mathrm{NMR}\left(\mathrm{CDCl}_{3}, 300 \mathrm{MHz}\right) \delta 7.88(\mathrm{~d}, 2 \mathrm{H}, J=$ $7.2 \mathrm{~Hz}), 7.66$ (t, $1 \mathrm{H}, J=7.2 \mathrm{~Hz}), 7.53(\mathrm{t}, 2 \mathrm{H}, J=7.2 \mathrm{~Hz}), 5.03(\mathrm{~s}, 1 \mathrm{H}), 4.68(\mathrm{~s}, 1 \mathrm{H})$, 3.77 (s, $2 \mathrm{H}), 1.87$ (s, $3 \mathrm{H}) .{ }^{13} \mathrm{C} \mathrm{NMR}\left(\mathrm{CDCl}_{3}, 75 \mathrm{MHz}\right) \delta$ 138.4, 133.6, 133.3, 128.9, $128.4,120.7,64.4,22.6$

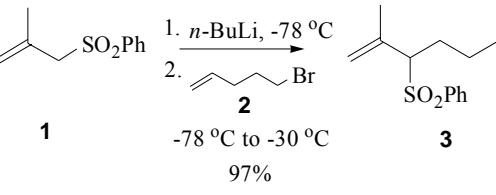

2-Methyl-3-phenylsulfonyl-1,7-octadiene (3) To a stirred solution containing 492 $\mathrm{mg}(3.0 \mathrm{mmol})$ of 2-methallyl phenyl sulfone in $50 \mathrm{~mL}$ of $\mathrm{THF}$ at $-78^{\circ} \mathrm{C}$ was slowly added $2.0 \mathrm{~mL}$ of $n$-BuLi (1.6 M in hexane) by syringe. After the solution had been stirred for $1 \mathrm{~h}$ at $-78{ }^{\circ} \mathrm{C}, 5$-bromo-1-pentene ( $460 \mathrm{mg}, 3.1 \mathrm{mmol}$ ) was added slowly by syringe. After the reaction mixture had been warmed up from $-78{ }^{\circ} \mathrm{C}$ to $-30{ }^{\circ} \mathrm{C}$ over 5 hours, it was diluted with saturated aqueous $\mathrm{NH}_{4} \mathrm{Cl}$ and extracted with ether $(50 \mathrm{~mL} \mathrm{x}$ 3). The combined organic layer was washed with brine, dried over $\mathrm{MgSO}_{4}$ and evaporated under reduced pressure. The residue was column chromatographed $(20 \%$ EtOAc in hexane) to yield $770 \mathrm{mg}$ (97\%) of 2-methyl-3-phenylsulfonyl-1,7-octadiene as a colorless oil. IR (neat) 3076, 2927, 1978, 1902, 1821, 1640, 1585, 1447, 1306, 1147, 1306, 1147, 912, 756. ${ }^{1} \mathrm{H} \mathrm{NMR}\left(\mathrm{CDCl}_{3}, 300 \mathrm{MHz}\right) \delta 7.85(\mathrm{~d}, 2 \mathrm{H}, J=7.2 \mathrm{~Hz})$, $7.62(\mathrm{t}, 1 \mathrm{H}, J=7.2 \mathrm{~Hz}), 7.52(\mathrm{t}, 2 \mathrm{H}, J=7.2 \mathrm{~Hz}), 5.74(\mathrm{~m}, 1 \mathrm{H}), 5.02(\mathrm{~s}, 1 \mathrm{H}), 4.96$ (m, $2 \mathrm{H}), 4.71(\mathrm{~s}, 1 \mathrm{H}), 3.55\left(\mathrm{dd}, 1 \mathrm{H}, J_{1}=11.7 \mathrm{~Hz}, J_{2}=3.5 \mathrm{~Hz}\right), 2.13-1.86(\mathrm{~m}, 4 \mathrm{H})$, $1.78(\mathrm{~s}, 3 \mathrm{H}), 1.45-1.25(\mathrm{~m}, 2 \mathrm{H}) .{ }^{13} \mathrm{C} \mathrm{NMR}\left(\mathrm{CDCl}_{3} 75 \mathrm{MHz}\right) \delta 137.7,137.6,136.8$, 
133.4, 129.0, 128.7, 120.3, 115.2, 72.4, 33.2, 25.9, 25.1, 20.1. MS (EI) $\mathrm{m} / \mathrm{z}$ (relative intensity) 264 (5), 123 (68), 81 (100), 77 (43), 67 (68), 55 (35). HRMS (EI) calcd for $\mathrm{C}_{9} \mathrm{H}_{15}\left(\mathrm{M}^{+}-\mathrm{SO}_{2} \mathrm{Ph}\right): 123.1179$, found 123.1174 .

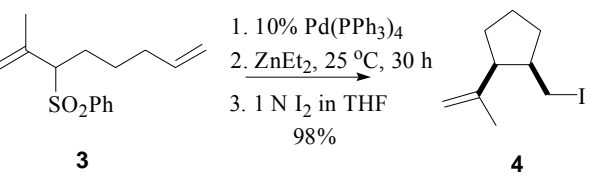

cis-1-Iodomethyl-2-isopropenylcyclopentane (4) To a solution of 3 (92 mg, $0.35 \mathrm{mmol}$ )

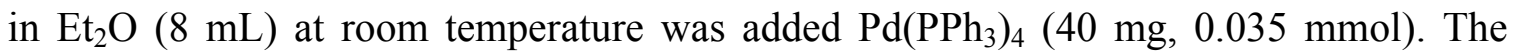
resulting solution was stirred for 5 min before $\mathrm{Et}_{2} \mathrm{Zn}(2.1 \mathrm{~mL}, 2.1 \mathrm{mmol}, 1.0 \mathrm{M}$ solution in hexane) was added. The resulting mixture was stirred for $30 \mathrm{~h}$ at room temperature before it was cooled to $0{ }^{\circ} \mathrm{C}$ and quenched with $\mathrm{I}_{2}(1.2 \mathrm{~g}, 4.7 \mathrm{mmol})$ in $5 \mathrm{~mL}$ of THF. The resulting mixture was stirred at room temperature for 30 min, diluted with $\mathrm{Et}_{2} \mathrm{O}$ (50 $\mathrm{mL}$ ), washed with $20 \% \mathrm{Na}_{2} \mathrm{~S}_{2} \mathrm{O}_{3}(10 \mathrm{~mL} \times 2)$, dried over $\mathrm{MgSO}_{4}$, filtered through cotton, and evaporated in vacuo. The residue was purified by flash-column chromatography to afford $85 \mathrm{mg}$ of 4 (98\% yield). IR (neat) 3081, 2956, 3872, 1646, 1450, 1174, 891. ${ }^{1} \mathrm{H}$ NMR $\left(\mathrm{CDCl}_{3}, 500 \mathrm{MHz}\right) \delta 4.88(\mathrm{~s}, 1 \mathrm{H}), 4.73(\mathrm{~s}, 1 \mathrm{H}), 3.15$ (dd, CHHI, J = 3.7, $\left.9.7 \mathrm{~Hz}\right)$, 2.80 (dd, CHHI, J = 9.7, 11.5 Hz), 2.54 (m, 1 H), 2.48 (m, 1 H), 1.94 (m, 1 H), 1.77 (s, 3 H), 1.76-1.6 (m, $5 \mathrm{H}) ;{ }^{13} \mathrm{C} \mathrm{NMR}\left(\mathrm{CDCl}_{3}, 75 \mathrm{MHz}\right) \delta 144.7,111.3,51.0,44.5,32.0,27.2$, 23.5, 22.1, 11.1; MS (EI) $\mathrm{m} / \mathrm{z}$ (relative intensity) $250\left(\mathrm{M}^{+}, 10\right), 123(50), 81$ (100), 67 (75), 55 (38). HRMS (EI) calcd for $\mathrm{C}_{9} \mathrm{H}_{15} \mathrm{I}\left(\mathrm{M}^{+}\right)$: 250.0220, found 250.0219.

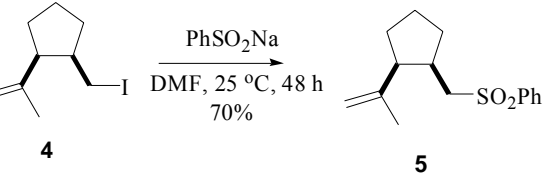


cis-1-(Benzenesulfonylmethyl)-2-(2-propenyl)cyclopentane $(5)^{13}$ To a stirred solution of 4 (500 mg, $2.0 \mathrm{mmol})$ in dry DMF $(25 \mathrm{~mL})$ was added benzene sulfinic acid sodium salt $(985 \mathrm{mg}, 6 \mathrm{mmol})$. The mixture was stirred under argon for 48 hours at room temperature after which the reaction mixture was diluted with $\mathrm{Et}_{2} \mathrm{O}(100 \mathrm{~mL})$ and washed with water $(5 \times 25 \mathrm{~mL})$. The organic layer was dried $\left(\mathrm{MgSO}_{4}\right)$ and filtered through cotton. Flash chromatography (12\% EtOAc in hexanes) provided the cis sulfone as a colorless liquid (370 mg, 70\% yield). ${ }^{1} \mathrm{H} \mathrm{NMR}\left(\mathrm{CDCl}_{3}, 300 \mathrm{MHz}\right) \delta$ 7.98-7.85 (m, $\left.2 \mathrm{H}\right), 7.7-7.5(\mathrm{~m}$, $3 \mathrm{H}) 4.84(\mathrm{~s}, 1 \mathrm{H}), 4.65(\mathrm{~s}, 1 \mathrm{H}), 2.95\left(\mathrm{dd}, 1 \mathrm{H}, \mathrm{J}=1.9,14.1 \mathrm{~Hz}, \mathrm{CHHSO}_{2} \mathrm{Ph}\right), 2.76(\mathrm{dd}, 1$ $\left.\mathrm{H}, \mathrm{J}=10.3,14.1 \mathrm{~Hz} \mathrm{CHHSO}_{2} \mathrm{Ph}\right), 2.5-2.6(\mathrm{~m}, 1 \mathrm{H}), 2.4-2.5(\mathrm{~m}, 1 \mathrm{H}), 1.57(\mathrm{~s}, 3 \mathrm{H}), 1.4-$ $1.9(\mathrm{~m}, 6 \mathrm{H}) ;{ }^{13} \mathrm{C} \mathrm{NMR}\left(\mathrm{CDCl}_{3}, 75 \mathrm{MHz}\right)$ 144.6, 139.9, 133.5, 129.2, 128.0, 112.1, 56.7, $50.9,35.6,30.6,27.2,22.9,22.1$.

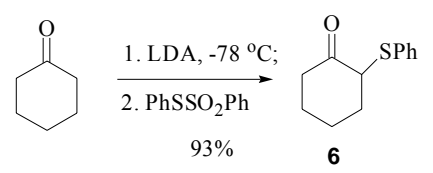

2-(Phenylthio)cyclohexanone (6) To a stirred solution of THF $(5 \mathrm{~mL})$ at $-78{ }^{\circ} \mathrm{C}$ was added LDA (1.1 mL, $2.2 \mathrm{mmol}, 2 \mathrm{M}$ solution in THF) followed by the addition of cyclohexanone (196 mg, $2.00 \mathrm{mmol})$ in $2 \mathrm{~mL}$ of THF. The resulting mixture was stirred at $-78{ }^{\circ} \mathrm{C}$ for half an hour before the temperature of the reaction mixture was raised to 0 ${ }^{\circ} \mathrm{C}$. After the mixture had been stirred at $0{ }^{\circ} \mathrm{C}$ for $10 \mathrm{~min}$, the temperature was cooled to $-78{ }^{\circ} \mathrm{C} . \mathrm{PhSSO}_{2} \mathrm{Ph}(600 \mathrm{mg}, 2.40 \mathrm{mmol})$ in $5 \mathrm{~mL}$ of THF was added dropwise to the reaction mixture. The mixture was stirred for $2 \mathrm{~h}$ further at this temperature before 10 $\mathrm{mL}$ of saturated $\mathrm{NaHCO}_{3}$ was added. The resulting mixture was extracted with $\mathrm{Et}_{2} \mathrm{O}$ (15 $\mathrm{mL} \times 3$ ). The combined organic layer was dried over $\mathrm{MgSO}_{4}$, filtered through cotton and evaporated in vacuo. The residue was purified by flash-column chromatography to 
afford $380 \mathrm{mg}$ of 6 (92\% yield). ${ }^{1} \mathrm{H}$ NMR $\left(\mathrm{CDCl}_{3}, 300 \mathrm{MHz}\right) \delta$ 7.43-7.27 (m, $\left.5 \mathrm{H}\right), 3.85$ (t, $1 \mathrm{H}, \mathrm{J}=5.2 \mathrm{~Hz}), 2.90(\mathrm{~m}, 1 \mathrm{H}), 2.32-2.21(\mathrm{~m}, 2 \mathrm{H}), 2.15-2.00(\mathrm{~m}, 1 \mathrm{H}), 2.00-1.75(\mathrm{~m}$, $3 \mathrm{H}), 1.72-1.60(\mathrm{~m}, 1 \mathrm{H}) ;{ }^{13} \mathrm{C} \mathrm{NMR}\left(\mathrm{CDCl}_{3}, 75 \mathrm{MHz}\right) \delta 207.7,134.0,132.0,129.1$, $127.5,56.6,39.2,34.1,27.5,22.8$.

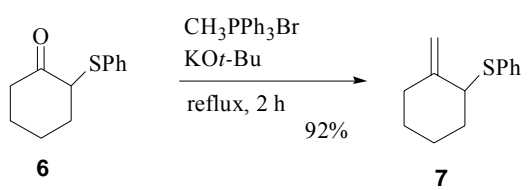

\section{1-(Phenylthio)-2-(methylene)cyclohexane (7)}

A mixture of methyltriphenylphosphonium bromide $(2.68 \mathrm{~g}, 7.50 \mathrm{mmol})$ and potassium $t$ butoxide $(842 \mathrm{mg}, 7.50 \mathrm{mmol})$ was placed in a three-neck flask $(100 \mathrm{~mL})$ equipped with a reflux condenser. THF $(40 \mathrm{~mL})$ was added and the resulting mixture was stirred at room temperature for $1 \mathrm{~h}$ before the dropwise addition of 2-(phenylthio)cyclohexanone $(1.0 \mathrm{~g}, 5.0 \mathrm{mmol})$ in $5 \mathrm{~mL}$ of THF via syringe. The reaction mixture was heated to reflux for $2 \mathrm{~h}$. After the reaction mixture was cooled to room temperature $\left(22{ }^{\circ} \mathrm{C}\right)$, the solution was concentrated in vacuo. Hexane was used to take the residue in the flask onto a silica gel column which was then eluted with hexane to give $949 \mathrm{mg}$ of 7 (93\% yield). ${ }^{1} \mathrm{H}$ NMR $\left(\mathrm{CDCl}_{3}, 300 \mathrm{MHz}\right) \delta$ 7.40-7.00 (m, $\left.5 \mathrm{H}\right), 4.67(\mathrm{~s}, 2 \mathrm{H}), 3.87(\mathrm{t}, 1 \mathrm{H}, \mathrm{J}=4.6 \mathrm{~Hz})$, $2.52(\mathrm{~m}, 1 \mathrm{H}), 2.10(\mathrm{~m}, 1 \mathrm{H}), 2.00-1.40(\mathrm{~m}, 6 \mathrm{H}) ;{ }^{13} \mathrm{C} \mathrm{NMR}\left(\mathrm{CDCl}_{3}, 75 \mathrm{MHz}\right) \delta 147.8$ 132.1, 128.8, 126.9, 109.7, 53.1, 33.6, 32.1, 28.1, 22.9.

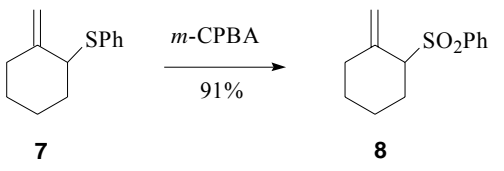




\section{1-(Benzenesulfonyl)-2-(methelene)cyclohexane (8)}

To a stirred solution of $7(204 \mathrm{mg}, 1.00 \mathrm{mmol})$ in $5 \mathrm{~mL}$ of $\mathrm{CH}_{2} \mathrm{Cl}_{2}$ at $0{ }^{\circ} \mathrm{C}$ was added $m$-CPBA (542 $\mathrm{mg}, 2.20 \mathrm{mmol}$ ) in $5 \mathrm{~mL}$ of $\mathrm{CH}_{2} \mathrm{Cl}_{2}$ in dropwise fashion via dropping pipet. The resulting mixture was stirred at $0{ }^{\circ} \mathrm{C}$ for $1 \mathrm{~h}$ before the addition of 10 $\mathrm{mL}$ of saturated $\mathrm{NaHCO}_{3}$. The reaction mixture was extracted with $\mathrm{CH}_{2} \mathrm{Cl}_{2}(10 \mathrm{~mL} \times 3)$. The combined organic layer was dried over $\mathrm{MgSO}_{4}$, filtered through cotton and evaporated in vacuo. The resulting residue was purified by flash-column chromatography to afford $214 \mathrm{mg}$ of 8 (91\% yield). IR (neat) 3127 (br), 2951, 2925, 2859, 1401, 922. ${ }^{1} \mathrm{H}$ NMR $\left(\mathrm{CDCl}_{3}, 300 \mathrm{MHz}\right) \delta$ 7.83-7.47 (m, $\left.5 \mathrm{H}\right), 4.88(\mathrm{~s}, 1 \mathrm{H}), 4.37$ (s, $1 \mathrm{H}), 3.65$ (m, $1 \mathrm{H}), 2.70-2.57(\mathrm{~m}, 2 \mathrm{H}), 2.22(\mathrm{~m}, 1 \mathrm{H}), 2.04-1.98$ (m, $1 \mathrm{H}), 1.87$ (m 1 $\mathrm{H}), 1.80-1.58(\mathrm{~m}, 2 \mathrm{H}), 1.35-1.27(\mathrm{~m}, 1 \mathrm{H}) ;{ }^{13} \mathrm{C} \mathrm{NMR}\left(\mathrm{CDCl}_{3}, 75 \mathrm{MHz}\right) \delta 140.4,137.9$, 133.5, 129.0, 128.8, 117.8, 68.2, 31.8, 26.8, 26.1, 21.4; MS (EI) m/z (relative intensity) $236\left(\mathrm{M}^{+}, 1.6\right), 143$ (11), 95 (100), 77 (40), 67 (45), 55 (26). HRMS (EI) calcd for $\mathrm{C}_{13} \mathrm{H}_{16} \mathrm{O}_{2} \mathrm{~S}\left(\mathrm{M}^{+}\right): 236.0871$, found 236.0870.

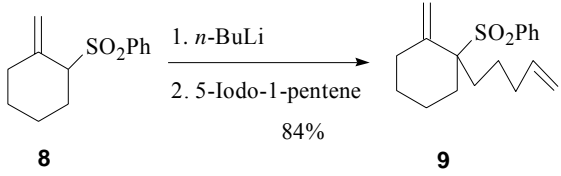

\section{(2-Methylene-1-pent-4-enylcyclohexanesulfonyl)benzene (9)}

To a stirred solution of $8(930 \mathrm{mg}, 3.94 \mathrm{mmol})$ in $\mathrm{THF}(30 \mathrm{~mL})$ at $-78{ }^{\circ} \mathrm{C}$ was added $n$-BuLi (2.7 mL, 4.3 mmol, 1.6 M solution in hexane). The resulting mixture was stirred at $-78{ }^{\circ} \mathrm{C}$ for $1 \mathrm{~h}$ before neat 1-iodo-4-pentene $(3.0 \mathrm{~mL}, 23.6 \mathrm{mmol})$ was added. After the resulting mixture was stirred at $-78{ }^{\circ} \mathrm{C}$ for $3 \mathrm{~h}$, the temperature was slowly raised to $-30{ }^{\circ} \mathrm{C}$ during a $0.5 \mathrm{~h}$ period and stirring at $-30{ }^{\circ} \mathrm{C}$ was continued for an 
additional $0.5 \mathrm{~h}$. Saturated $\mathrm{NaHCO}_{3}(20 \mathrm{~mL})$ was added to quench the reaction. The resulting mixture was extracted with $\mathrm{Et}_{2} \mathrm{O}(40 \mathrm{~mL} \times 3)$. The combined organic layer was dried over $\mathrm{MgSO}_{4}$, filtered through cotton, and evaporated in vacuo. The residue was purified by flash-column chromatography to afford $9(1.011 \mathrm{~g})$ in $84 \%$ yield. IR (neat) 3071, 2938, 2857, 1639, 1629, 1446, 1297, 1136, 1075, 912, 718, 691. ${ }^{1} \mathrm{H}$ NMR $\left(\mathrm{CDCl}_{3}\right.$, $300 \mathrm{MHz}) \delta$ 7.74-7.39 (m, 5 H), $5.62(\mathrm{~m}, 1 \mathrm{H}), 5.11(\mathrm{~s}, 1 \mathrm{H}), 4.92-4.83(\mathrm{~m}, 2 \mathrm{H}), 4.48(\mathrm{~s}$, $1 \mathrm{H}), 2.78(\mathrm{~m}, 1 \mathrm{H}), 2.37-2.23(\mathrm{~m}, 2 \mathrm{H}), 2.16-1.74(\mathrm{~m}, 5 \mathrm{H}), 1.68-1.55(\mathrm{~m}, 4 \mathrm{H}), 1.40-$ $1.17(\mathrm{~m}, 3 \mathrm{H}) ;{ }^{13} \mathrm{C} \mathrm{NMR}\left(\mathrm{CDCl}_{3}, 75 \mathrm{MHz}\right) \delta 141.1,137.9,135.2,133.5,130.8,128.2$, $118.3,115.3,70.5,34.2,33.8,33.7,29.3,25.7,22.8,22.2$; MS (EI) $\mathrm{m} / \mathrm{z}$ (relative intensity) 304 (M+1, 1), 266 (1), 163 (57), 121 (50), 109 (47), 95 (72), 81 (100), 67 (79), 55 (46). HRMS (EI) calcd for $\mathrm{C}_{18} \mathrm{H}_{24} \mathrm{O}_{2} \mathrm{~S}\left(\mathrm{M}^{+}\right)$: 304.1497 , found 304.1503 .

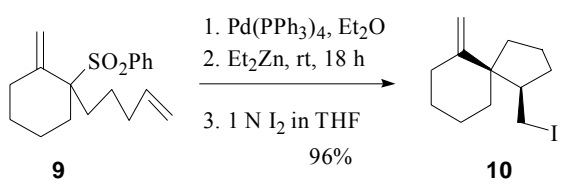

1-(Iodomethyl)-6-(methylene)spiro[4.5]decane (10) To a stirred solution of 9 (113 mg, $0.37 \mathrm{mmol})$ in $\mathrm{Et}_{2} \mathrm{O}(10 \mathrm{~mL})$ at room temperature were added $\mathrm{Pd}\left(\mathrm{PPh}_{3}\right)_{4}(21 \mathrm{mg}, 0.018$ mmol). The resulting solution was stirred for $5 \mathrm{~min}$ before $\mathrm{Et}_{2} \mathrm{Zn}(2.2 \mathrm{~mL}, 2.2 \mathrm{mmol}, 1.0$ M solution in hexane) was added. The resulting mixture was stirred for $18 \mathrm{~h}$ at room temperature before it was cooled to $0{ }^{\circ} \mathrm{C}$ and quenched with $\mathrm{I}_{2}(1.2 \mathrm{~g}, 4.7 \mathrm{mmol})$ in $5 \mathrm{~mL}$ of THF. After the resulting mixture was stirred at room temperature for $30 \mathrm{~min}$, it was diluted with $\mathrm{Et}_{2} \mathrm{O}(50 \mathrm{~mL})$, washed with $20 \% \mathrm{Na}_{2} \mathrm{~S}_{2} \mathrm{O}_{3}(10 \mathrm{~mL})$ and brine $(10 \mathrm{~mL})$, dried over $\mathrm{MgSO}_{4}$, filtered through cotton, and evaporated in vacuo. The residue was purified by flash-column chromatography to afford $103 \mathrm{mg}$ of $\mathbf{1 0}$ (96\% yield). IR (neat) 3081, 
2931, 2855, 1638, 1445, 1183, 895. ${ }^{1} \mathrm{H}$ NMR $\left(\mathrm{CDCl}_{3}, 300 \mathrm{MHz}\right) \delta 4.79(\mathrm{~s}, 1 \mathrm{H}), 4.63(\mathrm{~s}$, $1 \mathrm{H}), 3.16$ (ddd, $1 \mathrm{H}, \mathrm{J}=9.6,2.8,1.6 \mathrm{~Hz}), 2.70(\mathrm{dd}, 1 \mathrm{H}, \mathrm{J}=12.5,9.6 \mathrm{~Hz}), 2.52(\mathrm{~m}, 1 \mathrm{H})$, $2.28(\mathrm{~m}, 1 \mathrm{H}), 2.30-1.90(\mathrm{~m}, 2 \mathrm{H}), 1.90-1.48(\mathrm{~m}, 5 \mathrm{H}), 1.39-1.20(\mathrm{~m}, 3 \mathrm{H}), 1.12-1.02$ (m, $1 \mathrm{H}) ;{ }^{13} \mathrm{C} \mathrm{NMR}\left(\mathrm{CDCl}_{3}, 75 \mathrm{MHz}\right) \delta 152.2,108.2,53.9,45.6,38.2,35.5,33.8,29.6,28.8$, 22.7, 19.7, 14.2; MS (EI) m/z (relative intensity) $290\left(\mathrm{M}^{+}, 18\right), 163$ (100), 121 (71), 109 (63), 95 (85), 81 (87), 67 (62), 55 (37). HRMS (EI) calcd for $\mathrm{C}_{12} \mathrm{H}_{19} \mathrm{I}\left(\mathrm{M}^{+}\right)$: 290.0532 , found 290.0533 .

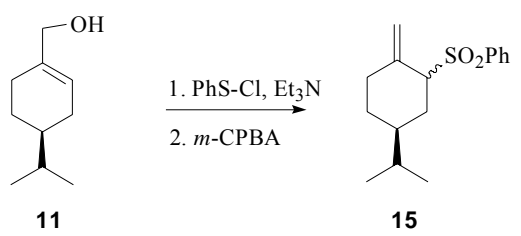

(5S)-5-isopropyl-2-methylene-1-phenylsulfonylcyclohexane (15) To a solution of allylic alcohol $11(371 \mathrm{mg}, 2.41 \mathrm{mmol})$ in $\mathrm{Et}_{3} \mathrm{~N}(1.34 \mathrm{~mL}, 9.6 \mathrm{mmol})$ was added a solution of benzenesulfenyl chloride ( $12 \mathrm{~mL}, 3.13 \mathrm{mmol}, 0.26 \mathrm{M}$ solution in benzene). The resulting solution was stirred at room temperature for $15 \mathrm{~min}$, then diluted with water, and extracted with ether $(30 \mathrm{~mL} \times 3)$. The combined organic layer, after being washed with saturated $\mathrm{NH}_{4} \mathrm{Cl}$ and brine, was dried over $\mathrm{MgSO}_{4}$, filtered through cotton and evaporated in vacuo. The residue was purified by flash-column chromatography to afford $560 \mathrm{mg}$ of allylic phenylsulfoxide $\mathbf{1 4}$ (89\% yield).

To the above phenylsulfoxide $14(560 \mathrm{mg}, 2.1 \mathrm{mmol})$ in $10 \mathrm{~mL}$ of $\mathrm{Et}_{2} \mathrm{O}$ at $-20{ }^{\circ} \mathrm{C}$ was added $\mathrm{m}$-CPBA (580 mg, $2.3 \mathrm{mmol}$ ). After the resulting mixture was stirred at -20 ${ }^{\circ} \mathrm{C}$ for $1 \mathrm{~h}$, Sat. $\mathrm{NaHCO}_{3}(10 \mathrm{~mL})$ was added to quench the reaction. The resulting mixture was extracted with $\mathrm{Et}_{2} \mathrm{O}(20 \mathrm{~mL} \times 3)$. The combined organic layer was dried over $\mathrm{MgSO}_{4}$, filtered through cotton and evaporated in vacuo. The residue was purified 
by flash-column chromatography to afford $15(501 \mathrm{mg})$ in $86 \%$ yield as two diastereomers with a ratio of 12.5:1. Spectra of major diasteomer: IR (neat) 3067, 2956, 2871, 1446, 1305, 1142, 1084. ${ }^{1} \mathrm{H}$ NMR $\left(\mathrm{CDCl}_{3}, 300 \mathrm{MHz}\right) \delta$ 7.91-7.31 (m, $\left.5 \mathrm{H}\right), 4.97$ (s, $1 \mathrm{H}), 4.44(\mathrm{~s}, 1 \mathrm{H}), 2.78(\mathrm{~m}, 1 \mathrm{H}), 3.77(\mathrm{~m}, 1 \mathrm{H}), 2.82-2.70(\mathrm{~m}, 1 \mathrm{H}), 2.63-2.57(\mathrm{~m}, 1$ H), 2.10-1.90 (m, 2 H), 1.54-1.42 (m, 2 H), 0.96 (d, 3 H, J = 3.5 Hz), 0.93 (d, 3 H, J = 3.5 $\mathrm{Hz}) ;{ }^{13} \mathrm{C} \mathrm{NMR}\left(\mathrm{CDCl}_{3}, 75 \mathrm{MHz}\right) \delta 140.4,137.9,133.0,129.0,128.8,117.8,68.8,37.8$, 32.6, 31.8, 29.8, 29.4, 19.8, 19.5; MS (EI) $\mathrm{m} / \mathrm{z}$ (relative intensity) $278\left(\mathrm{M}^{+}, 4\right), 137$ (83), 95 (72), 81 (100), 67 (36), 55 (32). HRMS (EI) calcd for $\mathrm{C}_{16} \mathrm{H}_{22} \mathrm{O}_{2} \mathrm{~S}\left(\mathrm{M}^{+}\right)$: 278.1341, found 278.1342 .

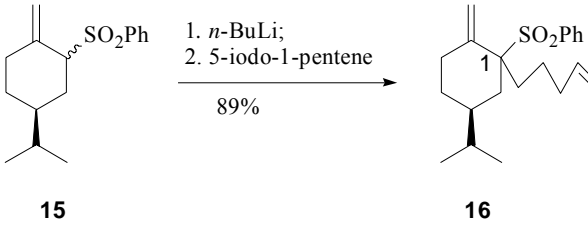

(5S)-5-isopropyl-2-methylene-1-(4-pentyl)-1-phenylsulfonylcyclohexane (16)

To a stirred solution of $\mathbf{1 5}(454 \mathrm{mg}, 1.63 \mathrm{mmol})$ in THF $(20 \mathrm{~mL})$ at $-78{ }^{\circ} \mathrm{C}$ was added $n$-BuLi (1.1 mL, 1.8 mmol, 1.6 M solution in hexane). The resulting mixture was stirred at $-78{ }^{\circ} \mathrm{C}$ for $1 \mathrm{~h}$ before neat 1 -iodo-4-pentene $(1.2 \mathrm{~mL}, 9.8 \mathrm{mmol})$ was added. After the resulting mixture was stirred at $-78{ }^{\circ} \mathrm{C}$ for $3 \mathrm{~h}$, the temperature was slowly raised to $-30{ }^{\circ} \mathrm{C}$ during a $0.5 \mathrm{~h}$ period and stirring at $-30{ }^{\circ} \mathrm{C}$ was continued for an additional $0.5 \mathrm{~h}$. Sat. $\mathrm{NaHCO}_{3}(20 \mathrm{~mL})$ was added to quench the reaction. The reaction mixture was extracted with $\mathrm{Et}_{2} \mathrm{O}(30 \mathrm{~mL} \times 3)$. The combined organic layer was dried over $\mathrm{MgSO}_{4}$, filtered through cotton, and evaporated in vacuo. The residue was purified by flash-column chromatography to afford $501 \mathrm{mg}$ of $\mathbf{1 6}$ (89\% yield) as two diatereomers in a ratio of 4:1. Spectra of major diatereomer: IR (neat) 3071, 2955, 2871, 1639, 1446, 
1298, 1140, 1082, 910, 721, 691. ${ }^{1} \mathrm{H}$ NMR $\left(\mathrm{CDCl}_{3}, 300 \mathrm{MHz}\right) \delta$ 7.83-7.49 (m, $\left.5 \mathrm{H}\right), 5.75$

(m, $1 \mathrm{H}), 5.18(\mathrm{~s}, 1 \mathrm{H}), 5.02-4.96(\mathrm{~m}, 2 \mathrm{H}), 2.22(\mathrm{~m}, 1 \mathrm{H}), 2.10-1.95(\mathrm{~m}, 5 \mathrm{H}), 1.82-1.73$ (m, 2 H), 1.64-1.57 (m, 3 H), 1.45 (m, 1 H), 1.31-1.25 (m, 2 H), 0.86 (d, 3 H, J = 2.5 Hz), $0.84(\mathrm{~d}, 3 \mathrm{H}, \mathrm{J}=2.4 \mathrm{~Hz}) ;{ }^{13} \mathrm{C} \mathrm{NMR}\left(\mathrm{CDCl}_{3}, 75 \mathrm{MHz}\right) \delta 142.6,138.1,135.7,133.5$, $131.2,128.3,116.8,115.3,70.9,37.3,34.3,34.0,32.9,31.7,26.7,23.6,19.8,19.4$; MS (EI) $\mathrm{m} / \mathrm{z}$ (relative intensity) $346\left(\mathrm{M}^{+}, 0.1\right), 278$ (0.2), $221(0.5), 205$ (51), 163 (17), 149 (83), 135 (62), 123 (73), 109 (97), 95 (99), 81 (100), 67 (85), 55 (46). HRMS (EI) calcd for $\mathrm{C}_{21} \mathrm{H}_{30} \mathrm{O}_{2} \mathrm{~S}\left(\mathrm{M}^{+}\right): 346.1967$, found 346.1956.

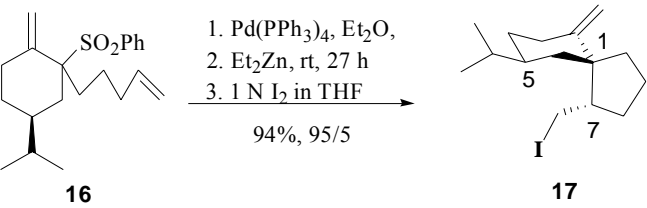

(1S), (9S)-1-Iodomethyl-9-isopropyl-6-methylene-spiro[4.5]decane (17)

To a stirred solution of $\mathbf{1 6}(101 \mathrm{mg}, 0.29 \mathrm{mmol})$ in $\mathrm{Et}_{2} \mathrm{O}(10 \mathrm{~mL})$ at room temperature were added $\mathrm{Pd}\left(\mathrm{PPh}_{3}\right)_{4}(17 \mathrm{mg}, 0.015 \mathrm{mmol})$. The resulting solution was stirred for $5 \mathrm{~min}$ before $\mathrm{Et}_{2} \mathrm{Zn}(1.7 \mathrm{~mL}, 1.7 \mathrm{mmol}, 1.0 \mathrm{M}$ solution in hexane) was added. The mixture was stirred for $27 \mathrm{~h}$ at room temperature before it was cooled to $0{ }^{\circ} \mathrm{C}$ and quenched with $\mathrm{I}_{2}(940 \mathrm{mg}, 3.7 \mathrm{mmol})$ in $5 \mathrm{~mL}$ of THF. After the resulting mixture was stirred at room temperature for $30 \mathrm{~min}$, it was diluted with $\mathrm{Et}_{2} \mathrm{O}(50 \mathrm{~mL})$. The organic layer, after being washed with $20 \% \mathrm{Na}_{2} \mathrm{~S}_{2} \mathrm{O}_{3}(15 \mathrm{~mL})$ and brine $(15 \mathrm{~mL})$, was dried over $\mathrm{MgSO}_{4}$, filtered through cotton, and evaporated in vacuo. The residue was purified by flash-column chromatography to afford $89 \mathrm{mg}$ of $\mathbf{1 7}$ (94\% yield) as two diastereomers in a ratio of 95 : 5. Major diastereomer: IR (neat) 3080, 2955, 2872, 1640, 1443, 1184, 894. ${ }^{1} \mathrm{H}$ NMR $\left(500 \mathrm{MHz}, \mathrm{CDCl}_{3}\right) \delta 4.80(\mathrm{~s}, 1 \mathrm{H}), 4.65(\mathrm{~s}, 1 \mathrm{H}), 3.16(\mathrm{ddd}, 1 \mathrm{H}, \mathrm{J}=9.7,2.7,1.7$ 
Hz), 2.70 (dd, $1 \mathrm{H}, \mathrm{J}=12.5,9.7 \mathrm{~Hz}), 2.51(\mathrm{~m}, 1 \mathrm{H}), 2.33$ (dt, $1 \mathrm{H}, \mathrm{J}=13.0,3.8 \mathrm{~Hz}), 2.03-$ $1.90(\mathrm{~m}, 3 \mathrm{H}), 1.88-1.71(\mathrm{~m}, 4 \mathrm{H}), 1.43-1.35(\mathrm{~m}, 3 \mathrm{H}), 1.28-1.20(\mathrm{~m}, 1 \mathrm{H}), 1.04(\mathrm{~m}, 1 \mathrm{H})$, $0.88(\mathrm{~d}, 3 \mathrm{H}, \mathrm{J}=6.1 \mathrm{~Hz}), 0.87(\mathrm{~d}, 3 \mathrm{H}, \mathrm{J}=6.0 \mathrm{~Hz}), 0.82(\mathrm{t}, 1 \mathrm{H}, \mathrm{J}=12.4 \mathrm{~Hz}) ;{ }^{13} \mathrm{C} \mathrm{NMR}$ $\left(\mathrm{CDCl}_{3}, 75 \mathrm{MHz}\right) \delta 152.2,107.9,53.8,46.1,41.8,39.8,35.3,33.6,32.6,31.6,29.5,20.2$, 19.8, 19.7, 14.3; MS (EI) m/z (relative intensity) $332\left(\mathrm{M}^{+}, 7\right), 205$ (75), 149 (88), 135 (65), 123 (73), 109 (97), 95 (98), 81 (100), 67 (82), 55 (64). HRMS (EI) calcd for $\mathrm{C}_{15} \mathrm{H}_{25} \mathrm{I}$ $\left(\mathrm{M}^{+}\right)$: 332.1001, found 332.1004.

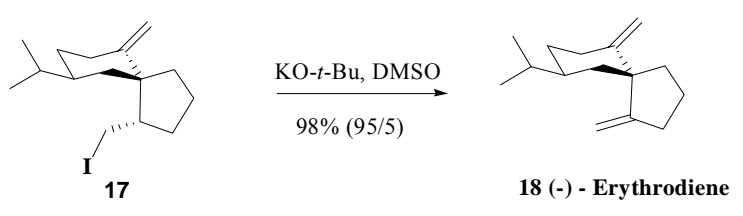

(-)-Erythrodiene (18) To a stirred solution of iodide $17(57 \mathrm{mg}, 0.17 \mathrm{mmol})$ in dry DMSO $(2 \mathrm{~mL})$ was added KO-tBu $(116 \mathrm{mg}, 1.0 \mathrm{mmol})$. Anhydrous $\mathrm{Et}_{2} \mathrm{O}(1 \mathrm{~mL})$ was added to generate a homogeneous solution. After the reaction mixture was stirred at room temperature for $30 \mathrm{~min}$, saturated $\mathrm{NH}_{4} \mathrm{Cl}(5 \mathrm{~mL})$ was added to quench the reaction. The resulting mixture was extracted with pentane $(10 \mathrm{~mL} \times 3)$. The combined pentane layer was dried over $\mathrm{MgSO}_{4}$, filtered through cotton and evaporated in cold to give (-)erythrodiene $(33 \mathrm{mg}$ ) in $95 \%$ yield as a $95: 5$ diastereomeric mixture. A sample of the major diastereomer was obtained by careful chromatography in minimal light with $\mathrm{AgNO}_{3}$ impregnated silica gel (Aldrich) $\left(5 \% \mathrm{Et}_{2} \mathrm{O}\right.$ in pentane). $[\alpha]_{\mathrm{D}}=-112^{\circ}(c=0.5$, $\left.\mathrm{CHCl}_{3}\right) . \quad$ IR (neat) $2956,2931,2871,1637,1443,891 .{ }^{1} \mathrm{H} \mathrm{NMR}\left(500 \mathrm{MHz}, \mathrm{CDCl}_{3}\right) \delta$ 4.98(s, $1 \mathrm{H}), 4.87$ (s, $1 \mathrm{H}), 4.77$ (s, $1 \mathrm{H}), 4.75$ (s, $1 \mathrm{H}), 2.50-2.33$ (m, 3 H), 2.28 (dt, $1 \mathrm{H}, \mathrm{J}$ = 13.6, $4.0 \mathrm{~Hz}$ ), $2.10(\mathrm{dt}, 1 \mathrm{H}, \mathrm{J}=12.3,7.1 \mathrm{~Hz}), 1.84-1.76(\mathrm{~m}, 2 \mathrm{H}), 1.70$ (quint, $2 \mathrm{H}, \mathrm{J}=$ $7.4 \mathrm{~Hz}), 1.61-1.38(\mathrm{~m}, 4 \mathrm{H}), 0.88(\mathrm{~d}, 3 \mathrm{H}, \mathrm{J}=6.7 \mathrm{~Hz}), 0.86(\mathrm{~d}, 3 \mathrm{H}, \mathrm{J}=6.6 \mathrm{~Hz}) ;{ }^{13} \mathrm{C} \mathrm{NMR}$ 
$\left(\mathrm{CDCl}_{3}, 75 \mathrm{MHz}\right) \delta 158.0,152.8,106.7,105.9,51.2,41.1,39.65,39.60,33.8,33.0,32.5$, 31.0, 20.7, 20.0, 19.6; MS (EI) m/z (relative intensity) $332\left(\mathrm{M}^{+}, 7\right), 205$ (75), 149 (88), 135 (65), 123 (73), 109 (97), 95 (98), 81 (100), 67 (82), 55 (64). HRMS (EI) calcd for $\mathrm{C}_{15} \mathrm{H}_{24}\left(\mathrm{M}^{+}\right): 204.1878$, found 204.1868.

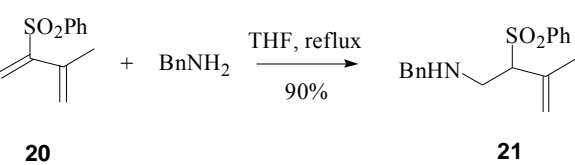

N-(2-Benzenesulfonyl-3-methyl-but-3-enyl)-benzylamine (21) To a three-neck flask equipped with a reflux condenser was added sequentially THF $(9 \mathrm{~mL}), 2$ benzenesulfonyl-3-methyl-1,3-butandiene $(170 \mathrm{mg}, 0.817 \mathrm{mmol})$ and benzylamine $(0.11$ $\mathrm{mL}, 1 \mathrm{mmol}$ ). The resulting mixture was heated at reflux for $12 \mathrm{~h}$. After the reaction mixture had cooled to room temperature, the solvent was evaporated and the residue was subjected to flash-column chromatography to give $232 \mathrm{mg}$ of $\mathbf{2 1}$ ( $90 \%$ yield). IR (neat) 3334 (br), 3062, 3028, 2922, 2849, 1447, 1305, 1145, 1084. ${ }^{1} \mathrm{H}$ NMR $\left(\mathrm{CDCl}_{3}\right) \delta$ 7.827.49 (m, 5 H), 7.31-7.20 (m, 5 H), 5.05 (s, 1 H), 4.73 (s, 1 H), 3.83 (dd, 1 H, J = 13.7, 7.1 $\mathrm{Hz}), 3.70(\mathrm{~d}, 2 \mathrm{H}, \mathrm{J}=5.3 \mathrm{~Hz}), 3.30(\mathrm{dd}, 1 \mathrm{H}, \mathrm{J}=12.5,5.5 \mathrm{~Hz}), 3.07(\mathrm{dd}, 1 \mathrm{H}, \mathrm{J}=12.5$, $8.4 \mathrm{~Hz}), 1.77(\mathrm{~s}, 3 \mathrm{H}) ;{ }^{13} \mathrm{C} \mathrm{NMR}\left(\mathrm{CDCl}_{3}\right) \delta 139.6,137.7,136.6,133.8,129.1,128.9$, 128.6, 128.2, 127.3, 120.5, 71.7, 53.7, 46.1, 21.0; MS (EI) $\mathrm{m} / \mathrm{z}$ (relative intensity) 315 $\left(\mathrm{M}^{+}, 2\right), 174$ (16), 120 (82), 91 (100), 77 (18), 65 (17). HRMS (EI) calcd for $\mathrm{C}_{18} \mathrm{H}_{21} \mathrm{NO}_{2} \mathrm{~S}$ $\left(\mathrm{M}^{+}\right)$: 315.1293, found 315.1297.

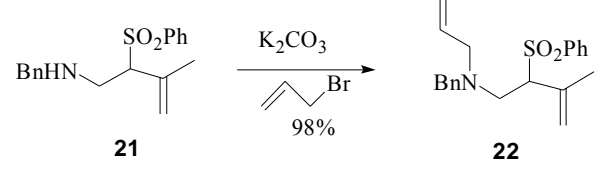


N-Allyl-(2-benzenesulfonyl-3-methyl-but-3-enyl)-benzyl-amine (22) To a stirred solution of phenyl sulfone $21(228 \mathrm{mg}, 0.723 \mathrm{mmol})$ in $\mathrm{CH}_{3} \mathrm{CN}(5 \mathrm{~mL})$ at $0{ }^{\circ} \mathrm{C}$ was added allyl bromide $(0.125 \mathrm{~mL}, 1.45 \mathrm{mmol})$ followed by $\mathrm{K}_{2} \mathrm{CO}_{3}(300 \mathrm{mg}, 2.17 \mathrm{mmol})$. The resulting mixture was stirred at room temperature for $14 \mathrm{~h}$. The solvent was evaporated and the residue was subjected to flash-column chromatography to give the title compound $22(253 \mathrm{mg})$ in 98\% yield. IR (neat) 3063, 3028, 2976, 2924, 2806, 1447, 1306, 1146, 1085. ${ }^{1} \mathrm{H} \mathrm{NMR}\left(\mathrm{CDCl}_{3}, 300 \mathrm{MHz}\right) \delta$ 7.84-7.49 (m, $\left.5 \mathrm{H}\right), 7.30-7.20(\mathrm{~m}, 5 \mathrm{H}), 5.76$ (m, 1 H), 5.14-5.09 (m, 2 H), $5.06(\mathrm{~s}, 1 \mathrm{H}), 4.72(\mathrm{~s}, 1 \mathrm{H}), 3.78(\mathrm{dd}, 1 \mathrm{H}, \mathrm{J}=9.1,5.2 \mathrm{~Hz}), 3.64$ $(\mathrm{d}, 1 \mathrm{H}, \mathrm{J}=13.5 \mathrm{~Hz}), 3.42(\mathrm{~d}, 1 \mathrm{H}, \mathrm{J}=13.5 \mathrm{~Hz}), 3.18-3.05(\mathrm{~m}, 3 \mathrm{H}), 2.93(\mathrm{dd}, 1 \mathrm{H}, \mathrm{J}=$ 14.1, 7.3 Hz), $1.78(\mathrm{~s}, 3 \mathrm{H}) ;{ }^{13} \mathrm{C} \mathrm{NMR}\left(\mathrm{CDCl}_{3}, 75 \mathrm{MHz}\right) \delta 138.7,138.4,137.0,135.0$, $133.7,129.1,129.0,128.4,127.3,120.7,118.4,70.8,58.5,57.3,50.3,20.5 ;$ MS (EI) $\mathrm{m} / \mathrm{z}$ (relative intensity) $355\left(\mathrm{M}^{+}, 2\right), 328$ (1), 214 (9), 160 (100), 91 (81), 77 (15), 65 (11). HRMS (EI) calcd for $\mathrm{C}_{21} \mathrm{H}_{25} \mathrm{NIO}_{2} \mathrm{~S}\left(\mathrm{M}^{+}\right)$: 355.1606, found 355.1593.

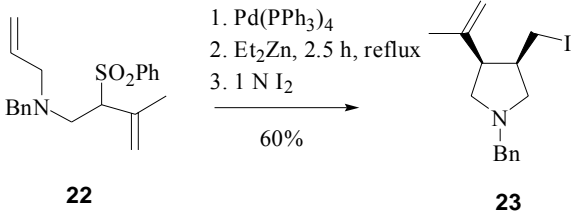

1-Benzyl-3-iodomethyl-4-isopropenyl-pyrrolidene (23) To a stirred solution of 22 (196 mg, $0.552 \mathrm{mmol})$ in $\mathrm{Et}_{2} \mathrm{O}(10 \mathrm{~mL})$ at room temperature was added $\mathrm{Pd}\left(\mathrm{PPh}_{3}\right)_{4}(64$ $\mathrm{mg}, 0.055 \mathrm{mmol})$. The resulting solution was stirred for $5 \mathrm{~min}$ before $\mathrm{Et}_{2} \mathrm{Zn}(5.5 \mathrm{~mL}, 5.5$ mmol, 1.0 M solution in hexane) was added. The mixture was heated to reflux and stirred for $2 \mathrm{~h} 15 \mathrm{~min}$ before it was cooled to $0{ }^{\circ} \mathrm{C}$ over 15 min and quenched with $\mathrm{I}_{2}(2.98$ $\mathrm{g}, 11.73 \mathrm{mmol}$ ) in $10 \mathrm{~mL}$ of THF. After the mixture was stirred for an additional $30 \mathrm{~min}$, it was diluted with ether $(50 \mathrm{~mL})$ and washed with $20 \% \mathrm{Na}_{2} \mathrm{~S}_{2} \mathrm{O}_{3}(20 \mathrm{~mL} \times 2)$. The 
organic layer was collected and dried over $\mathrm{MgSO}_{4}$, filtered, and evaporated in vacuo. The residue was purified by flash-column chromatography to afford 23 (113 $\mathrm{mg}$ ) in $60 \%$ yield along with $23 \mathrm{mg}$ of recovered starting material (68\% adjusted yield). IR (neat) 3062, 3026, 2960, 2924, 2791, 1452, 1147, 894, 749, 699. ${ }^{1} \mathrm{H}$ NMR $\left(\mathrm{CDCl}_{3}, 300 \mathrm{MHz}\right) \delta$ 7.36-7.25 (m, 5 H), 4.92 (s, 1 H), 4.76 (s, 1 H), 3.67 (s, 2 H), 3.16 (dd, 1 H, 7.6, 3.9 Hz), 3.20-3.15 (m, $1 \mathrm{H}), 2.98$ (dd, $1 \mathrm{H}, \mathrm{J}=11.5,9.6 \mathrm{~Hz}), 2.87-2.70$ (m, $3 \mathrm{H}), 2.63$ (dd, $1 \mathrm{H}, \mathrm{J}$ $=11.5,11.5 \mathrm{~Hz}), 2.44(\mathrm{dd}, 1 \mathrm{H}, \mathrm{J}=9.6,5.3 \mathrm{~Hz}), 1.77(\mathrm{~s}, 3 \mathrm{H}) ;{ }^{13} \mathrm{C} \mathrm{NMR}\left(\mathrm{CDCl}_{3}, 75\right.$ MHz) $\delta 142.52,139.2,128.7,128.4,127.1,112.7,61.9,60.5,56.5,49.0,43.2,23.8,9.9$; MS (EI) m/z (relative intensity) $341\left(\mathrm{M}^{+}, 10\right), 250$ (8), 214 (86), 125 (10), 91 (100), 65 (17), 55 (12). HRMS (EI) calcd for $\mathrm{C}_{15} \mathrm{H}_{20} \mathrm{NI}\left(\mathrm{M}^{+}\right)$: 341.0641, found 341.0655. 


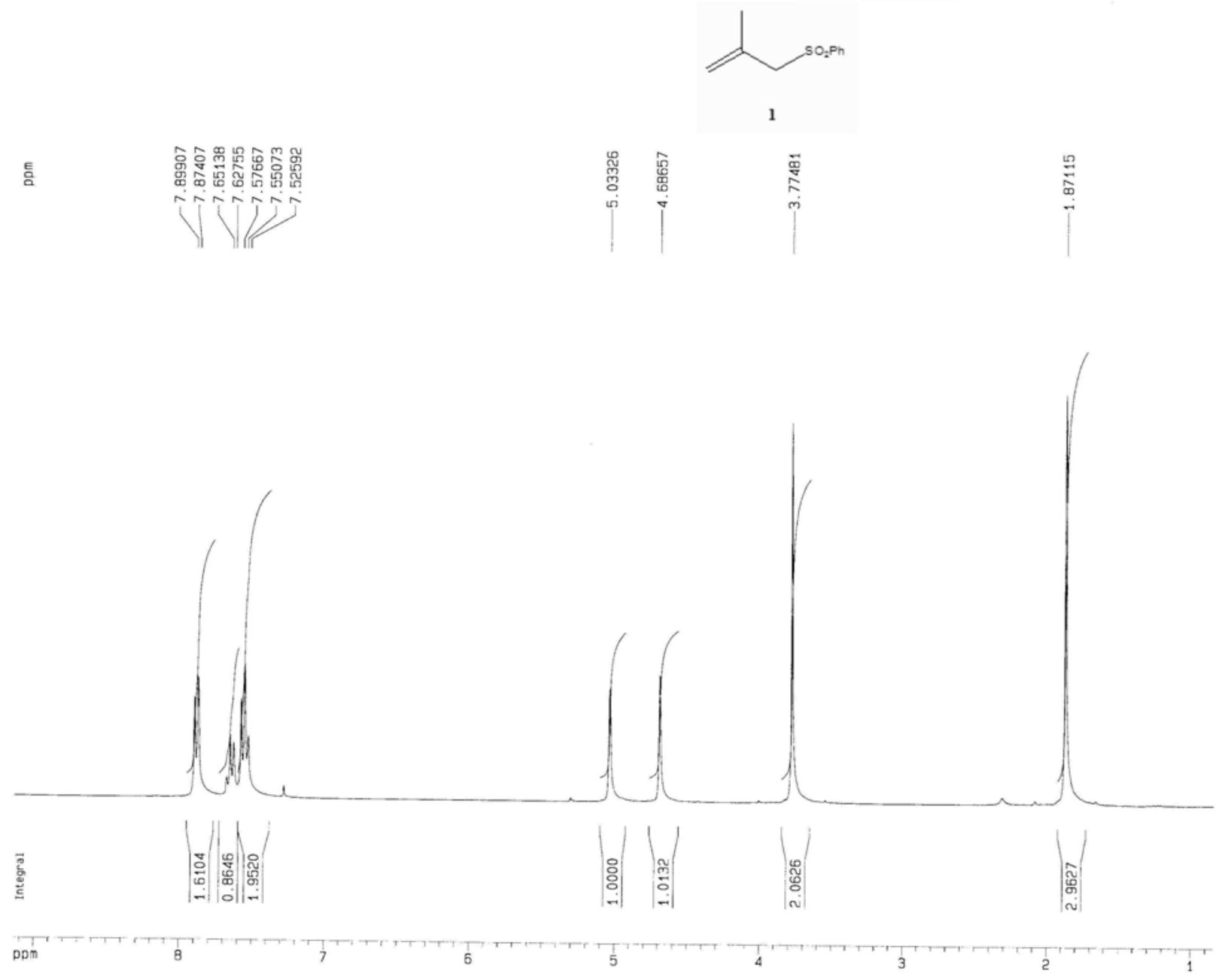




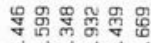

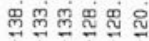

Sir

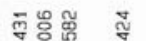

재용

V

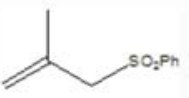

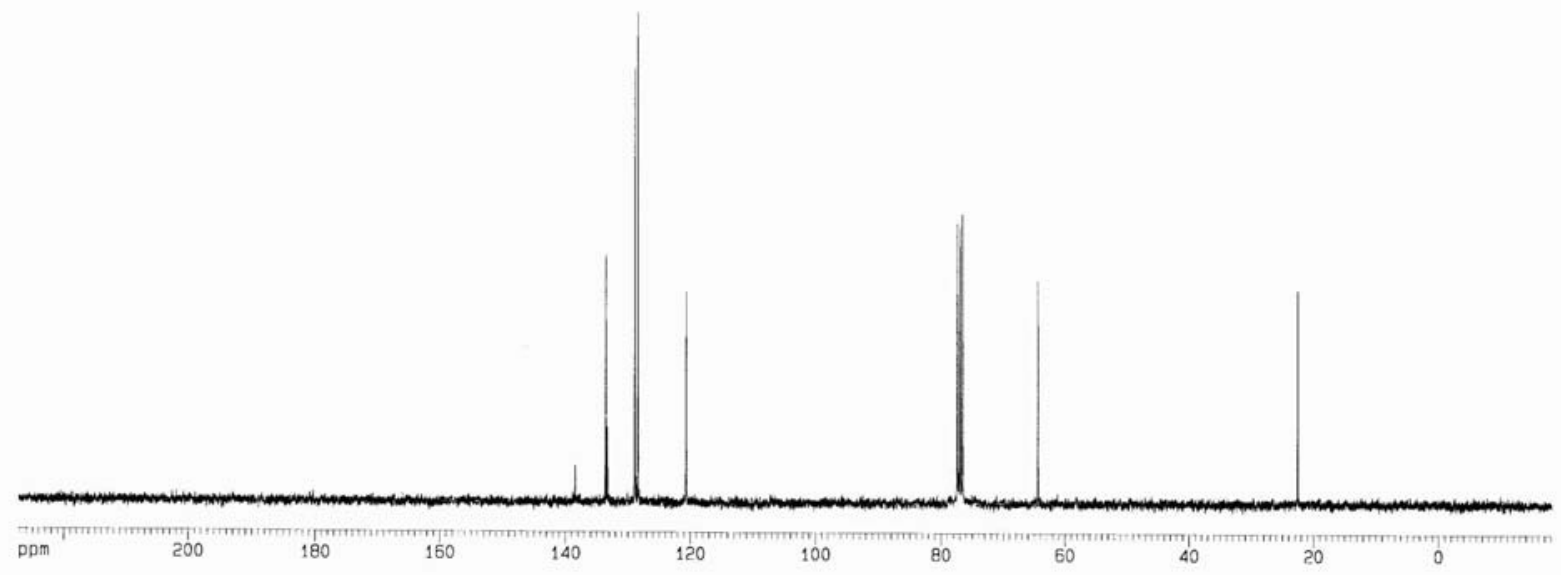




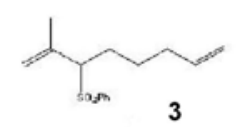

镸
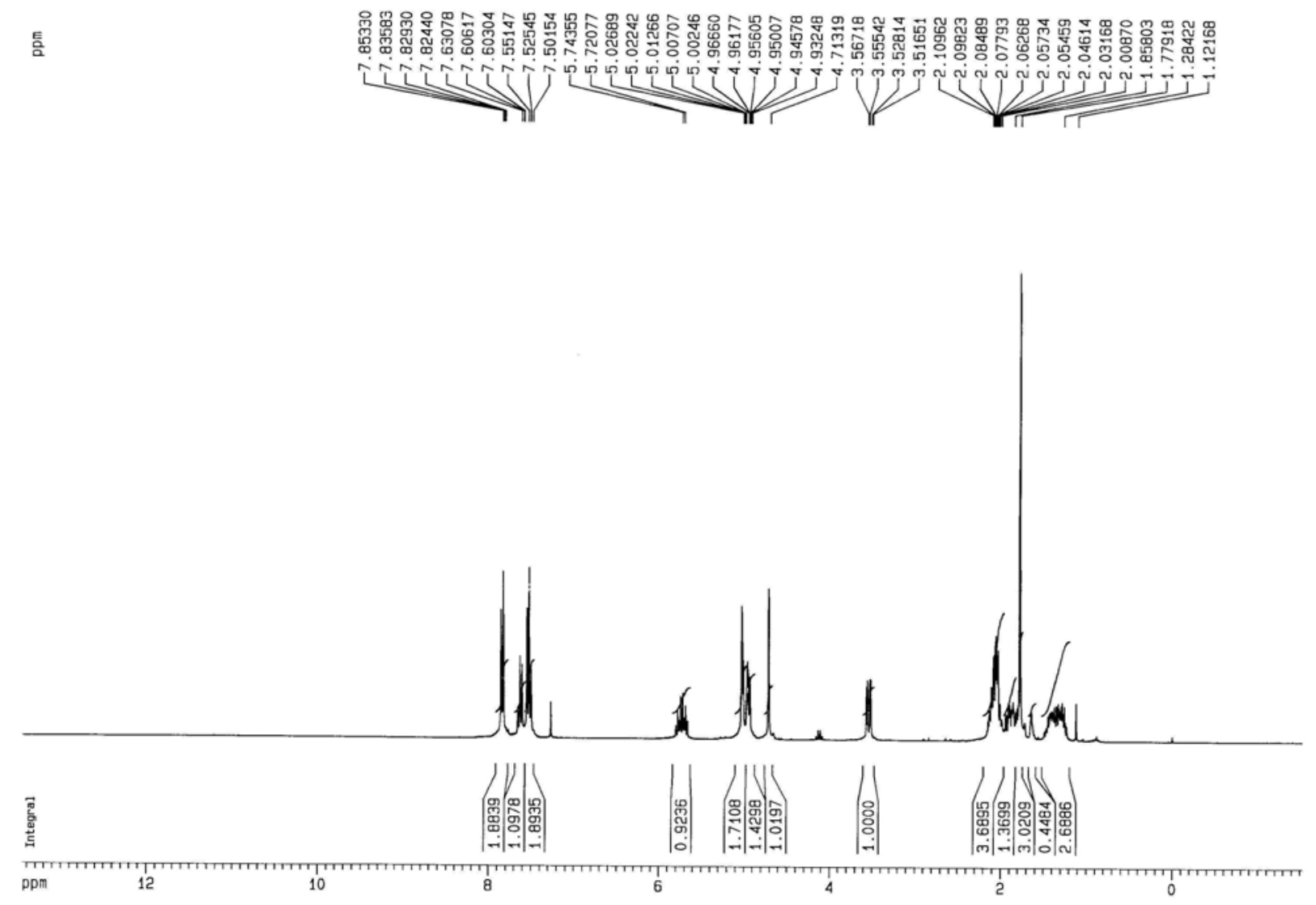

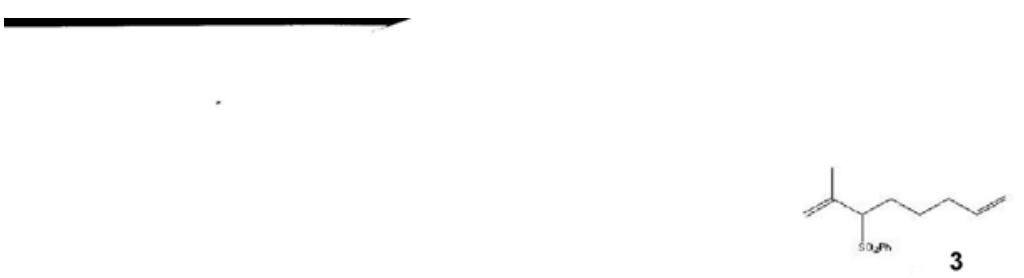

틈

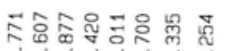

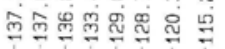

ชูํำ

रत्रूल

Фิ 兽哭

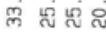

VI
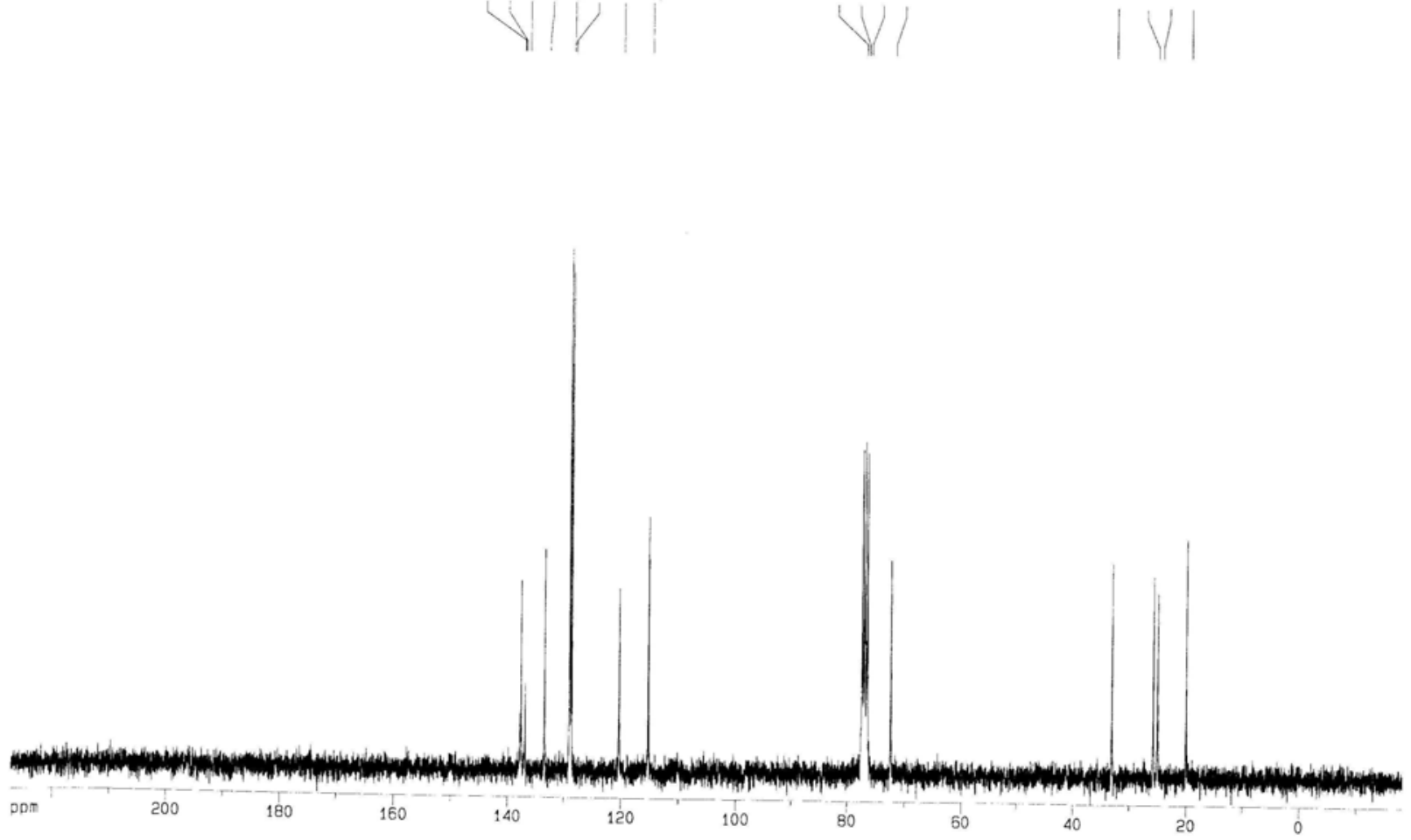

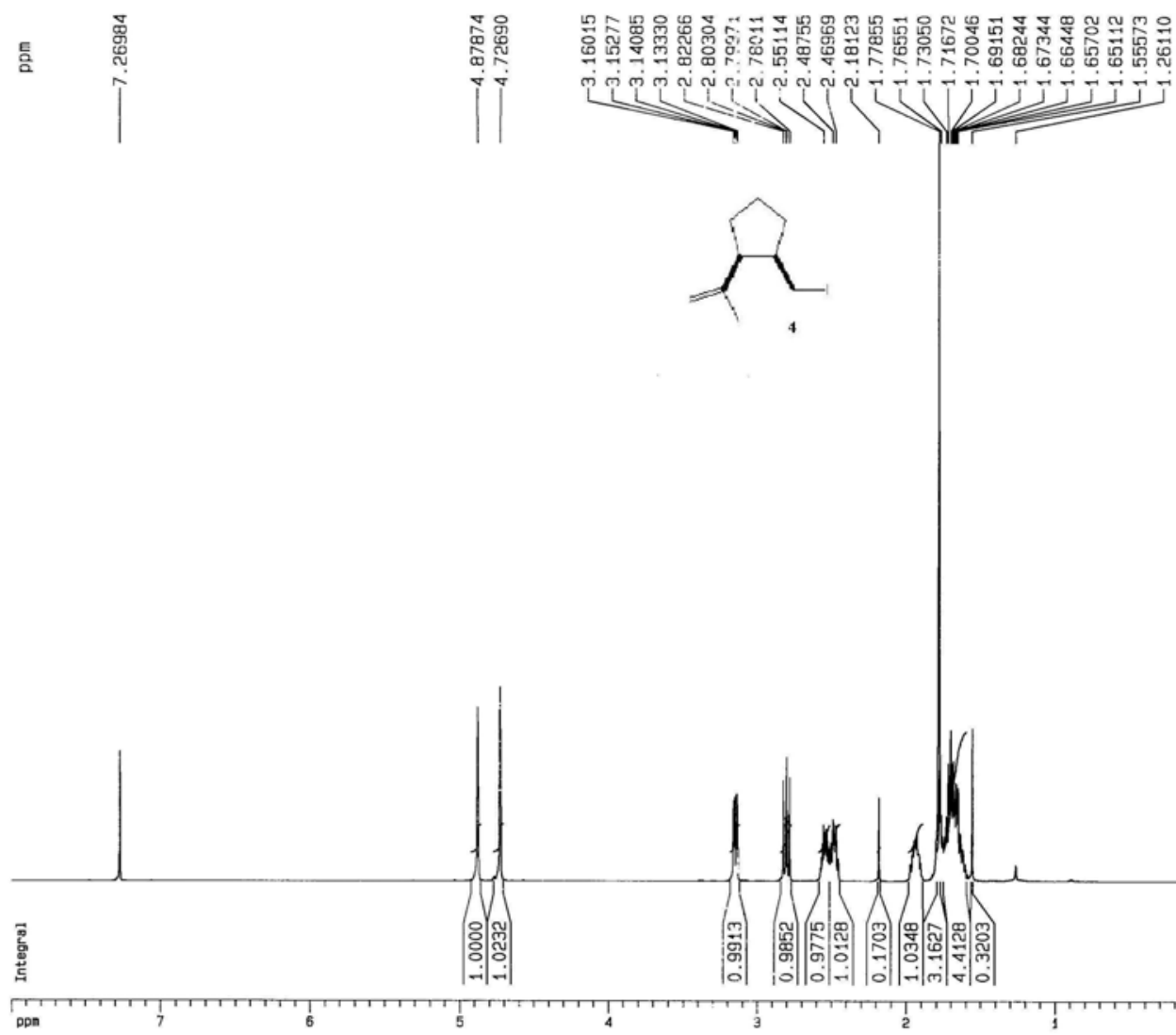

ร.

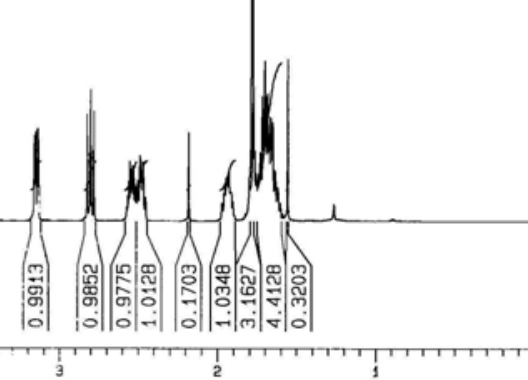

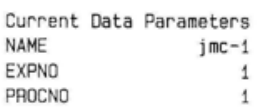

PAOCNO

F2 - Acquisition Parameters

Date_ $\quad 500000$

$\begin{array}{ll}\text { Time } & 10.41 \\ \text { INSTRUM } & \text { spect }\end{array}$

PAOBHD $5 \mathrm{~mm}$ TXI 13C

PULPROG 29

SOLVENT

NS

$\begin{array}{lr}\text { OS } & 0 \\ \text { SWH } & 7507.507 \mathrm{~Hz}\end{array}$

FIDRES $\quad 0.229111 \mathrm{~Hz}$

RG

DW

DE

D1

P1

DE

SF01

NUC1
PL1

2.1823988 sec 57

66.600 usec 6.00 usec $290.0 \mathrm{~K}$

12. $00000000 \mathrm{sec}$

10.00 usec

6.00 usec

$500.1330008 \mathrm{MHz}$

$0.00 \mathrm{~dB}$

F2 - Processing parameters

SI 32768

SF $\quad 500.1300238 \mathrm{MHz}$

WDW EM

$\begin{array}{lc}\text { SSB } & 0 \\ \text { LB } & 0.40 \mathrm{~Hz}\end{array}$

$\begin{array}{lr}\text { GB } & 0 \\ P C & 1.00\end{array}$

10 NMA plot parameters

CX $\quad 20.00 \mathrm{~cm}$

$\begin{array}{ll}F_{1} & 8.000 \mathrm{pp}\end{array}$

$\begin{array}{lr}\text { F1 } & 4001.04 \mathrm{~Hz} \\ \text { Fap } & 0.000 \mathrm{ppm}\end{array}$

F2 $\quad 0.00 \mathrm{~Hz}$

PPMCM $\quad 0.40000 \mathrm{pDm} / \mathrm{cm}$

$200.05202 \mathrm{~Hz} / \mathrm{cm}$ 

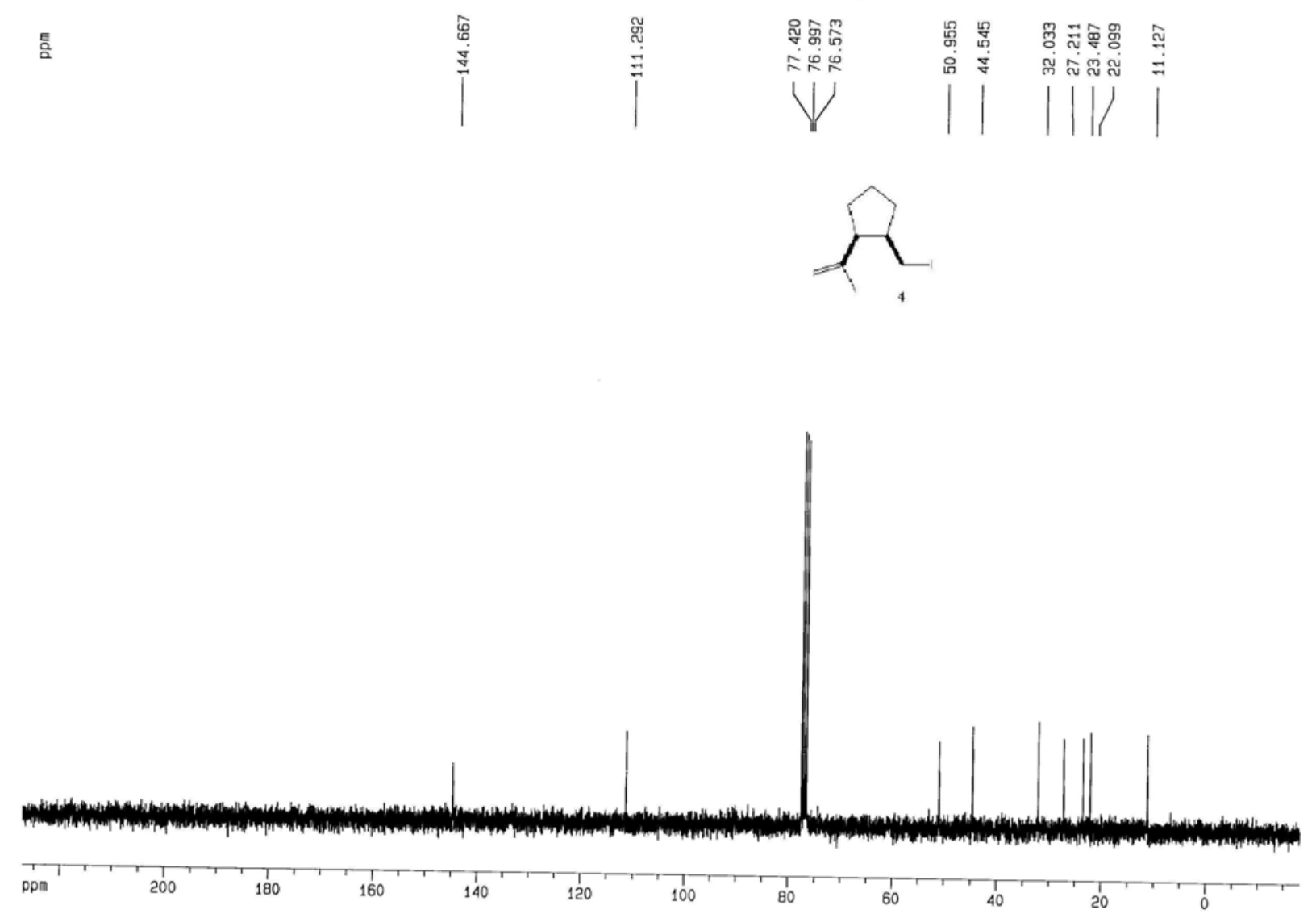
Cis sulfone $300 \mathrm{MHz} \mathrm{CDCl} 13 \mathrm{rt}$
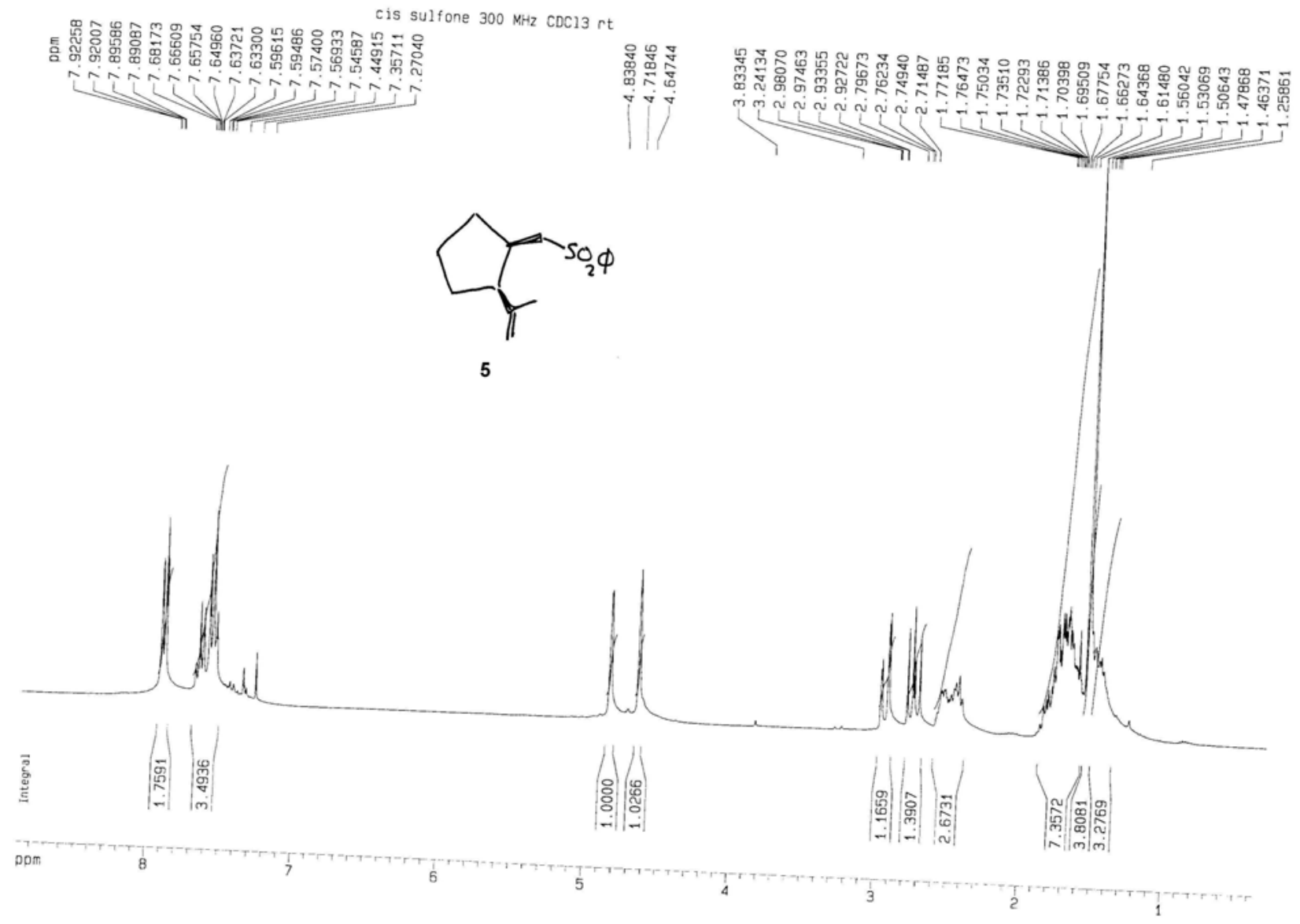

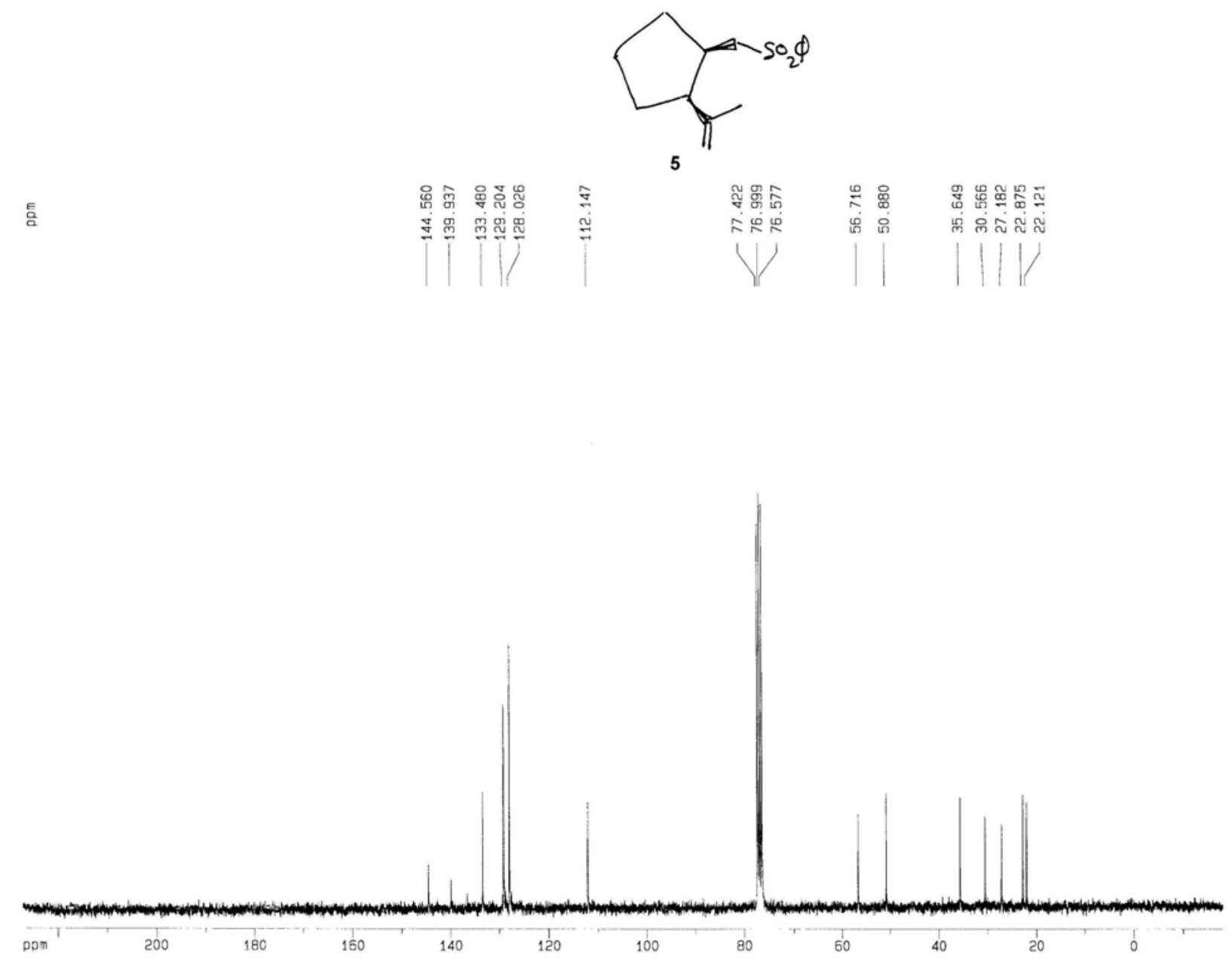
kai0708 check 2-Phenylthiocyclohexanone
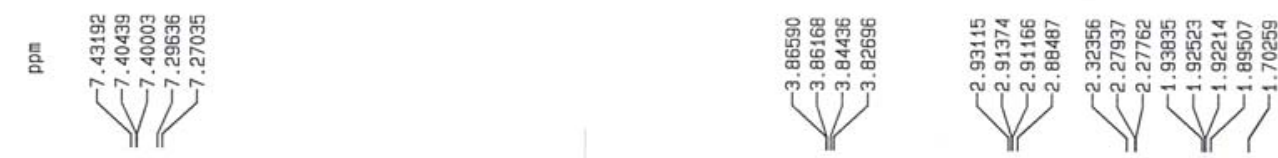

$\underbrace{\mathrm{O}}$

6
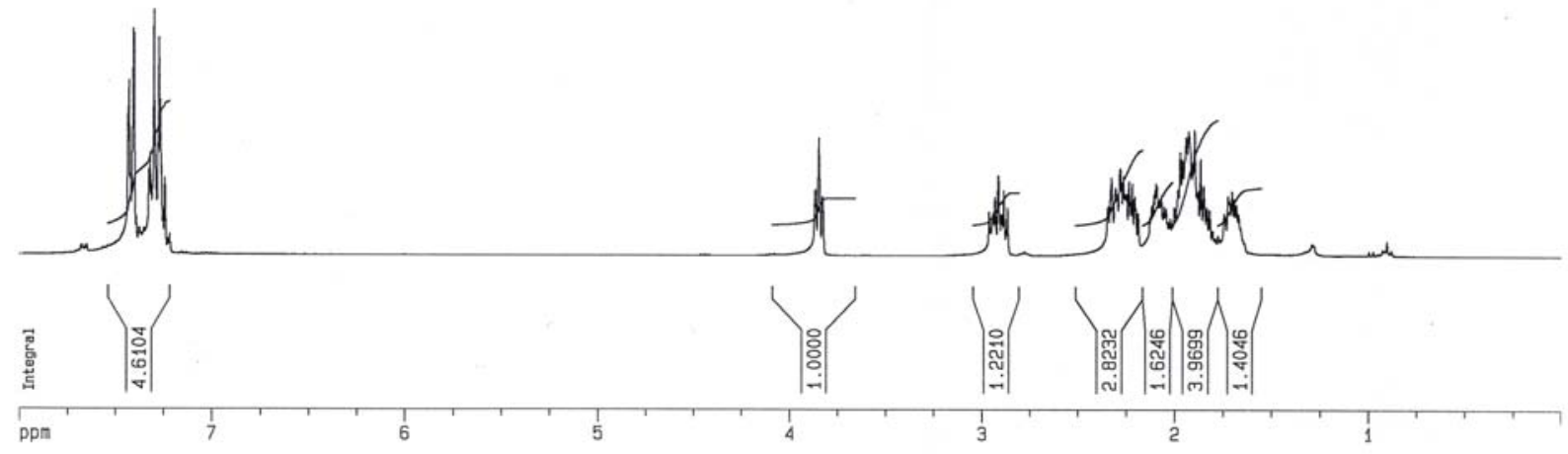


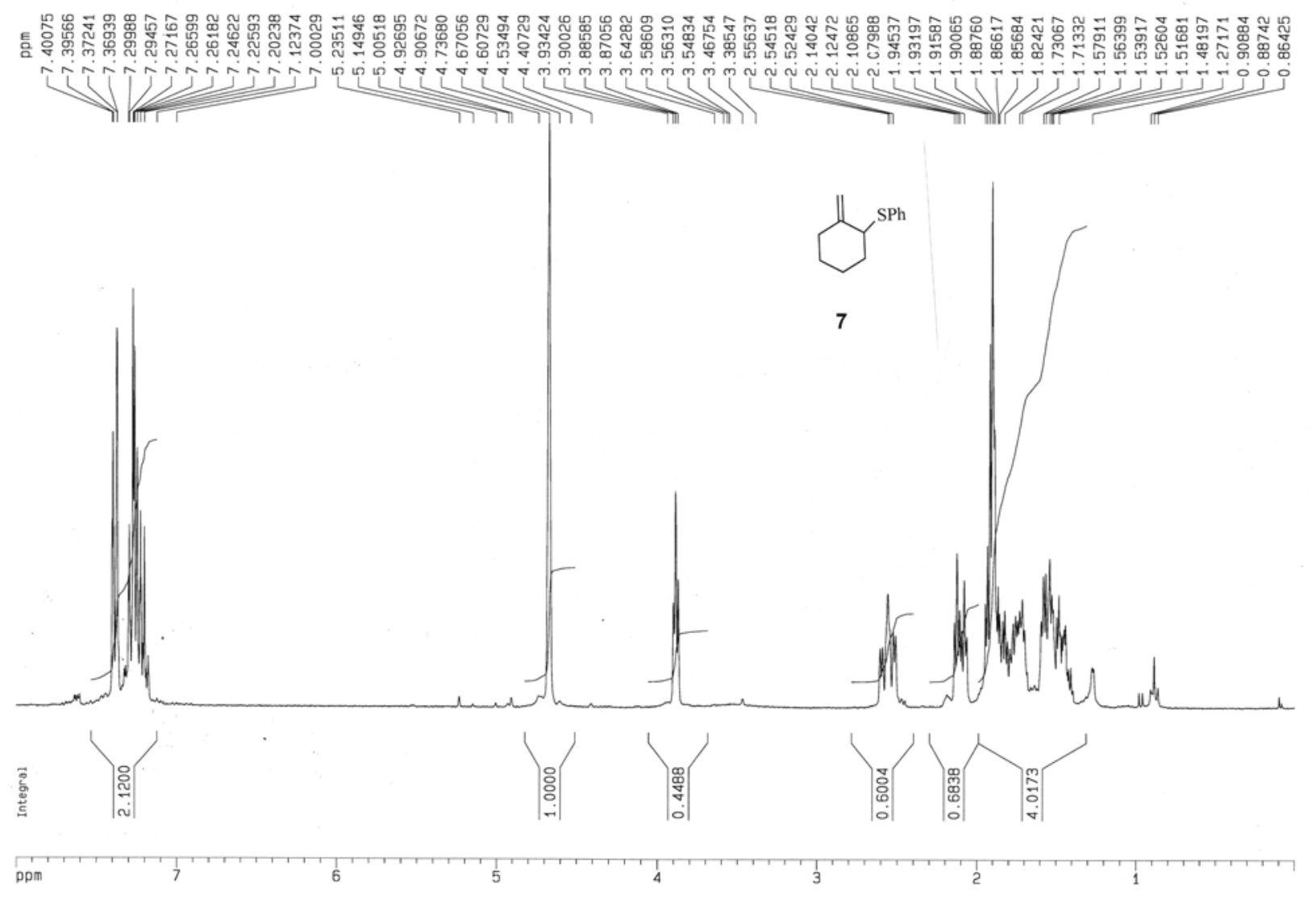




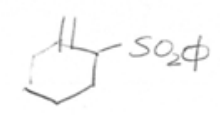
alfa-pheny lsul fonyl-1-methe lenecyclohexane
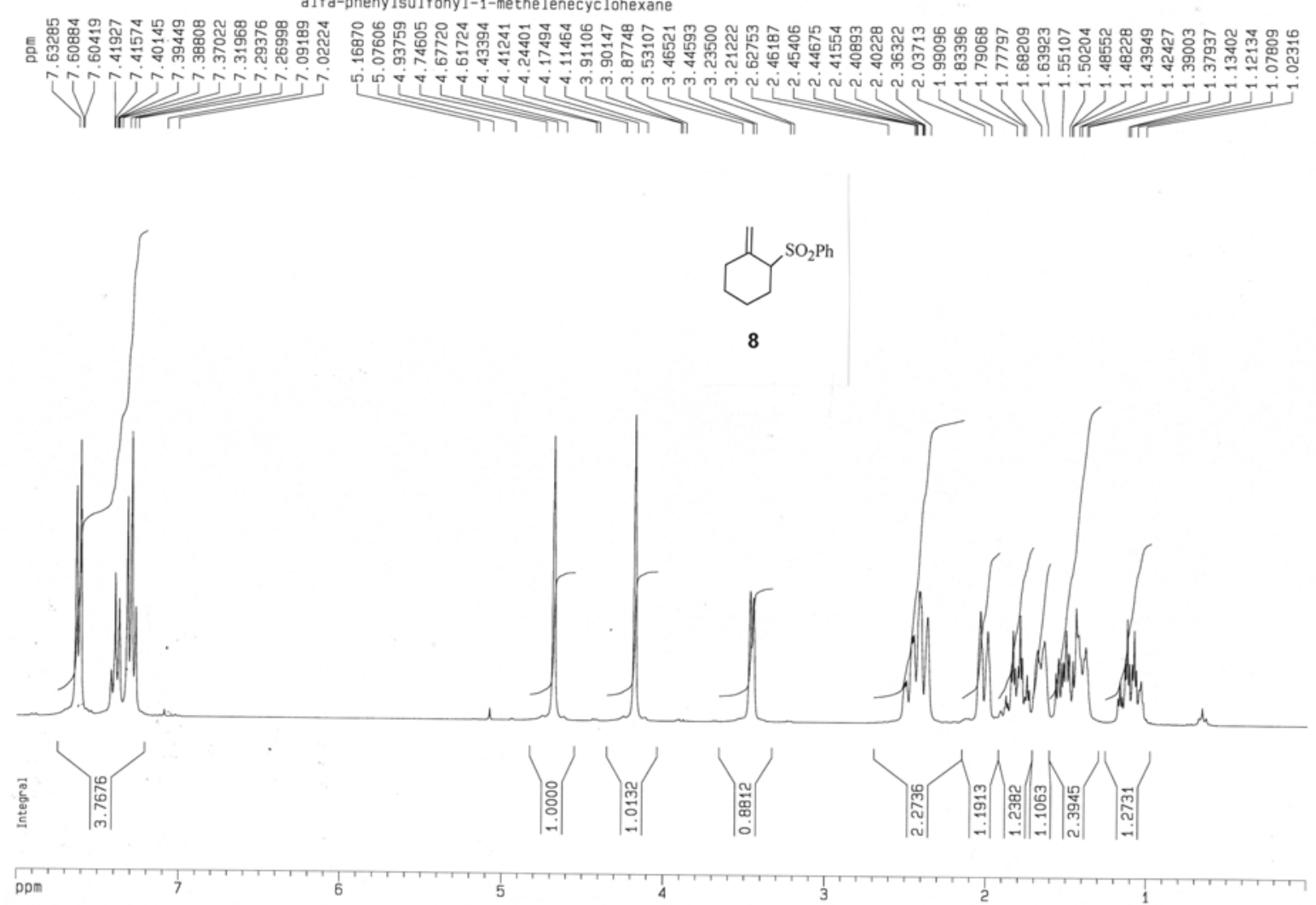


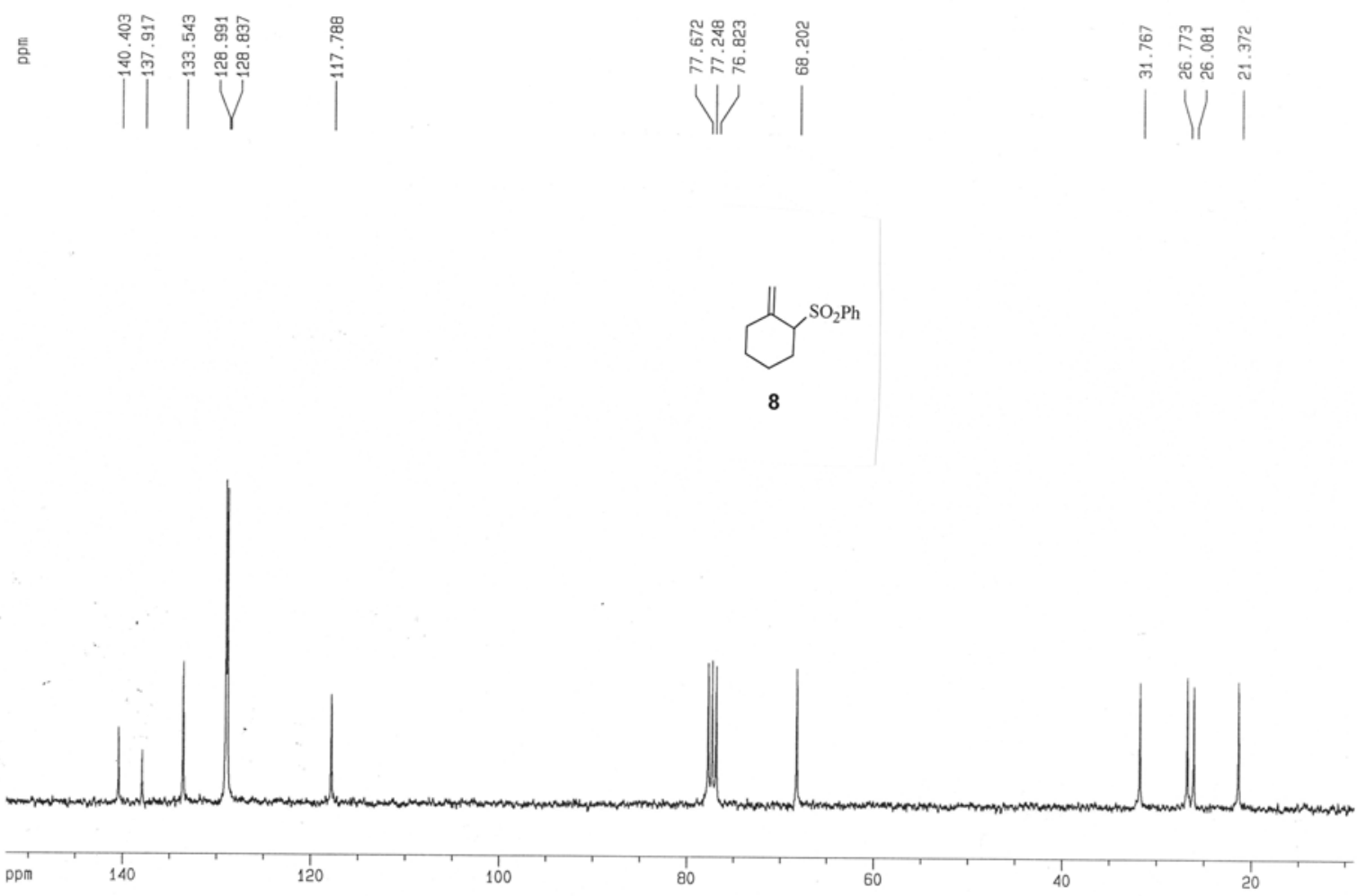




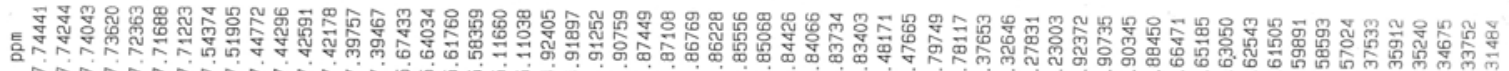

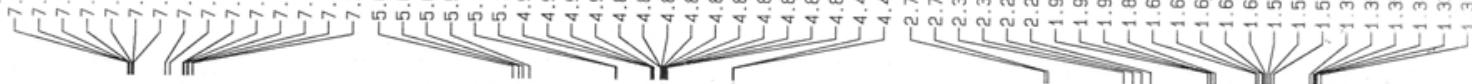<smiles>C=C1CCCCC1C1CCCC1</smiles>

9

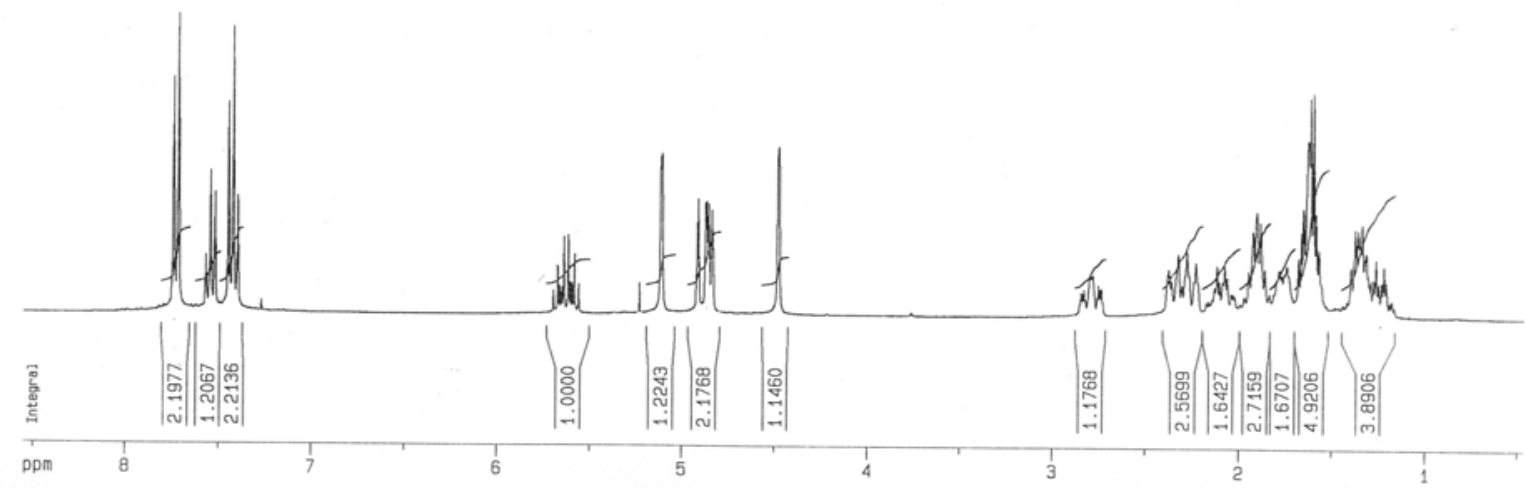




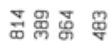

슝요

VI

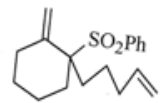

9

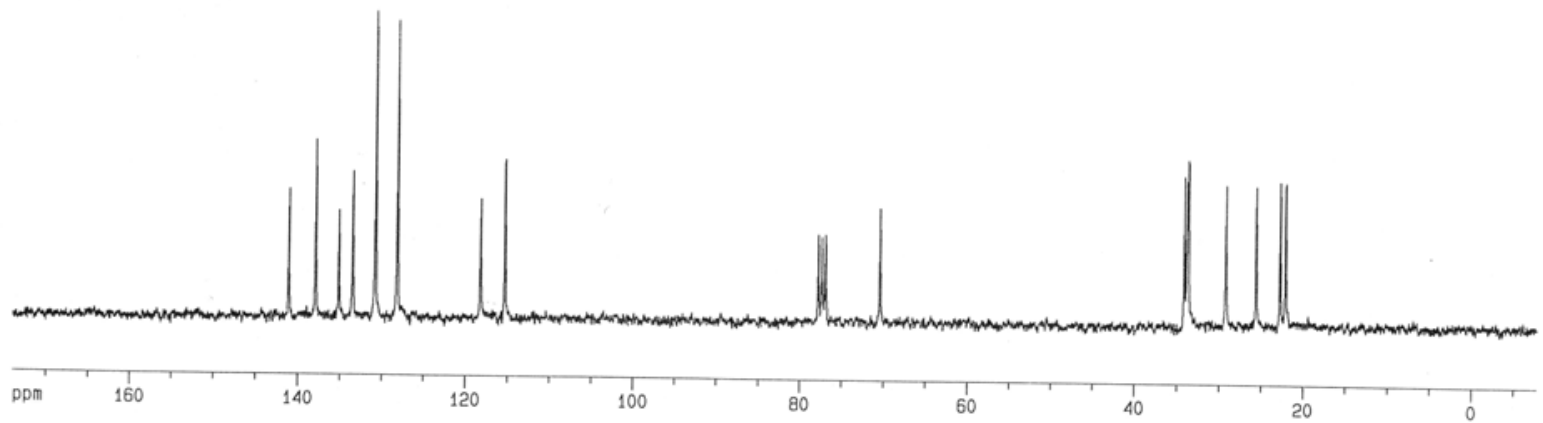


spirobicyle
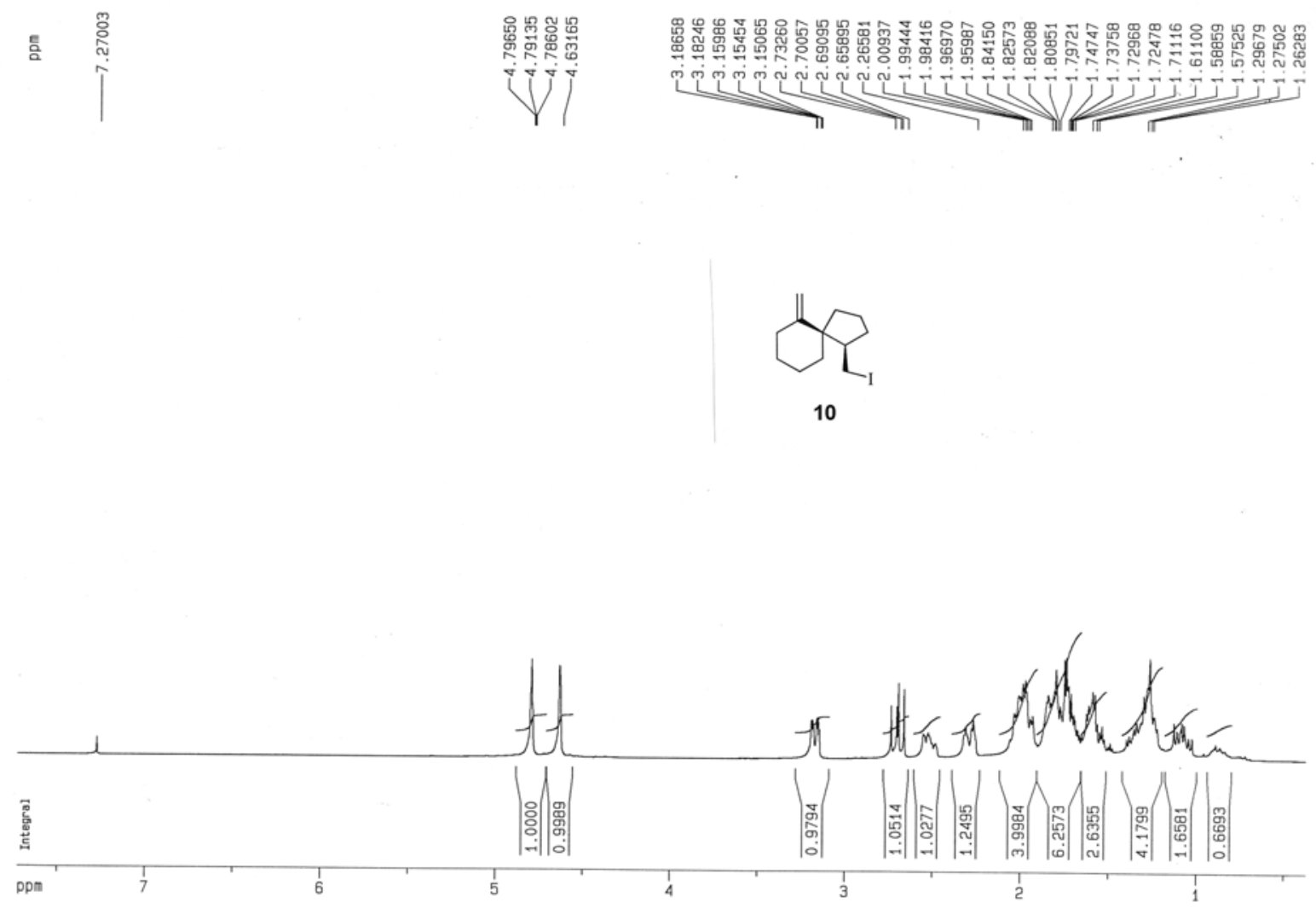
k0225 301b

言

ป

ณั
总贯舟

RE⿱

IV
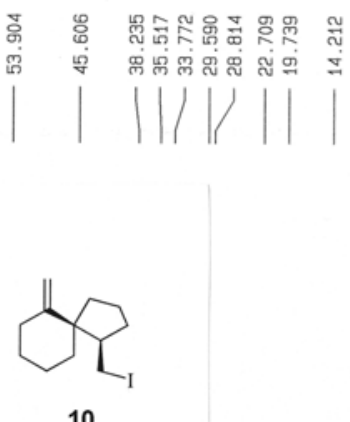

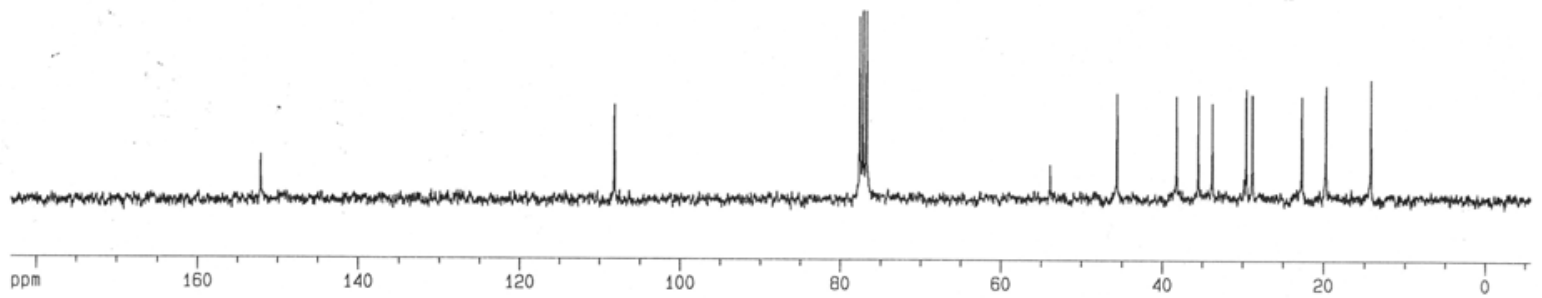




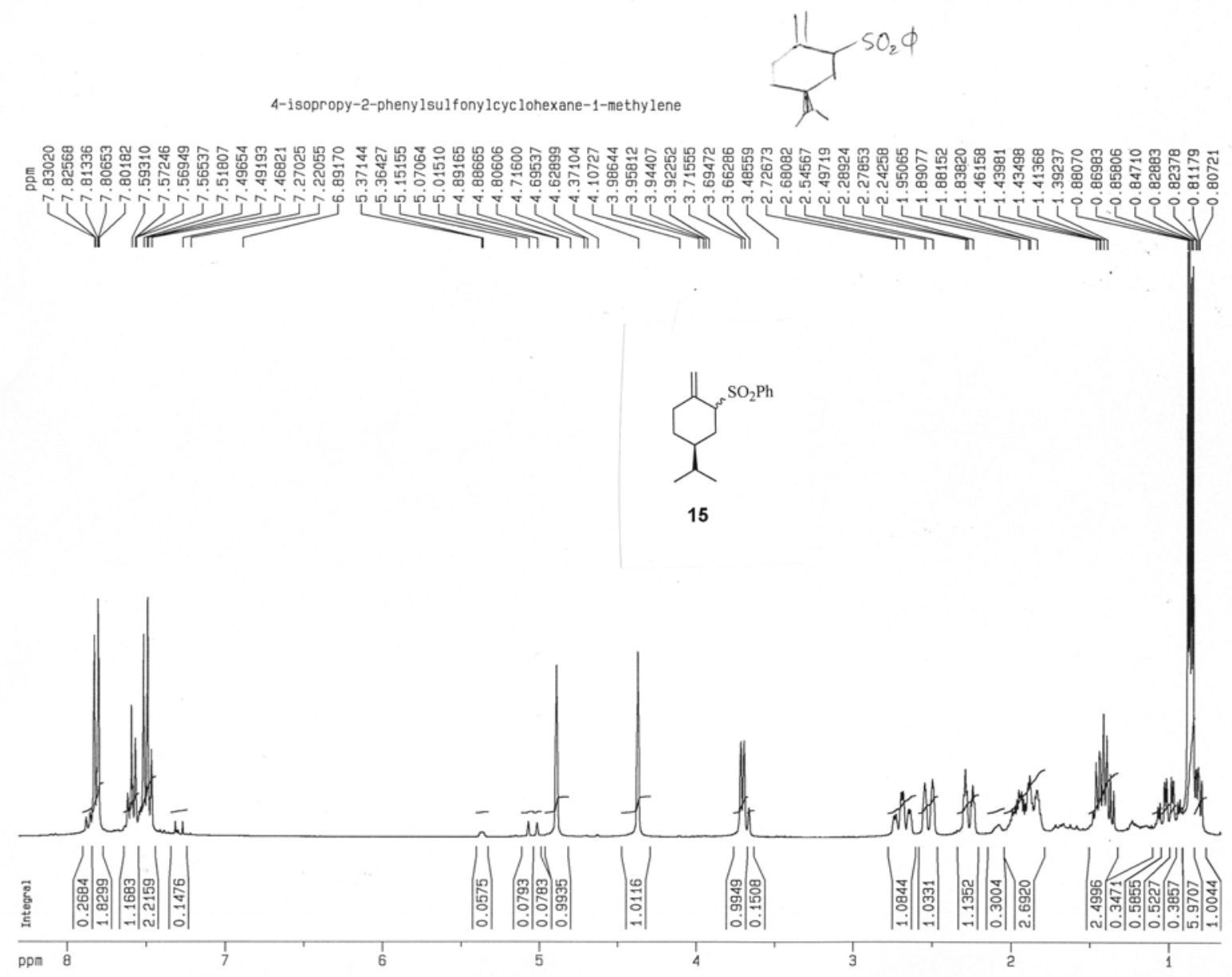



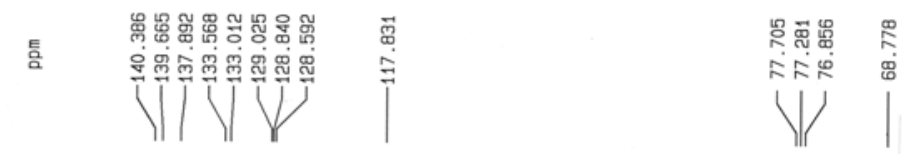

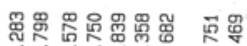

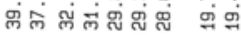

111111

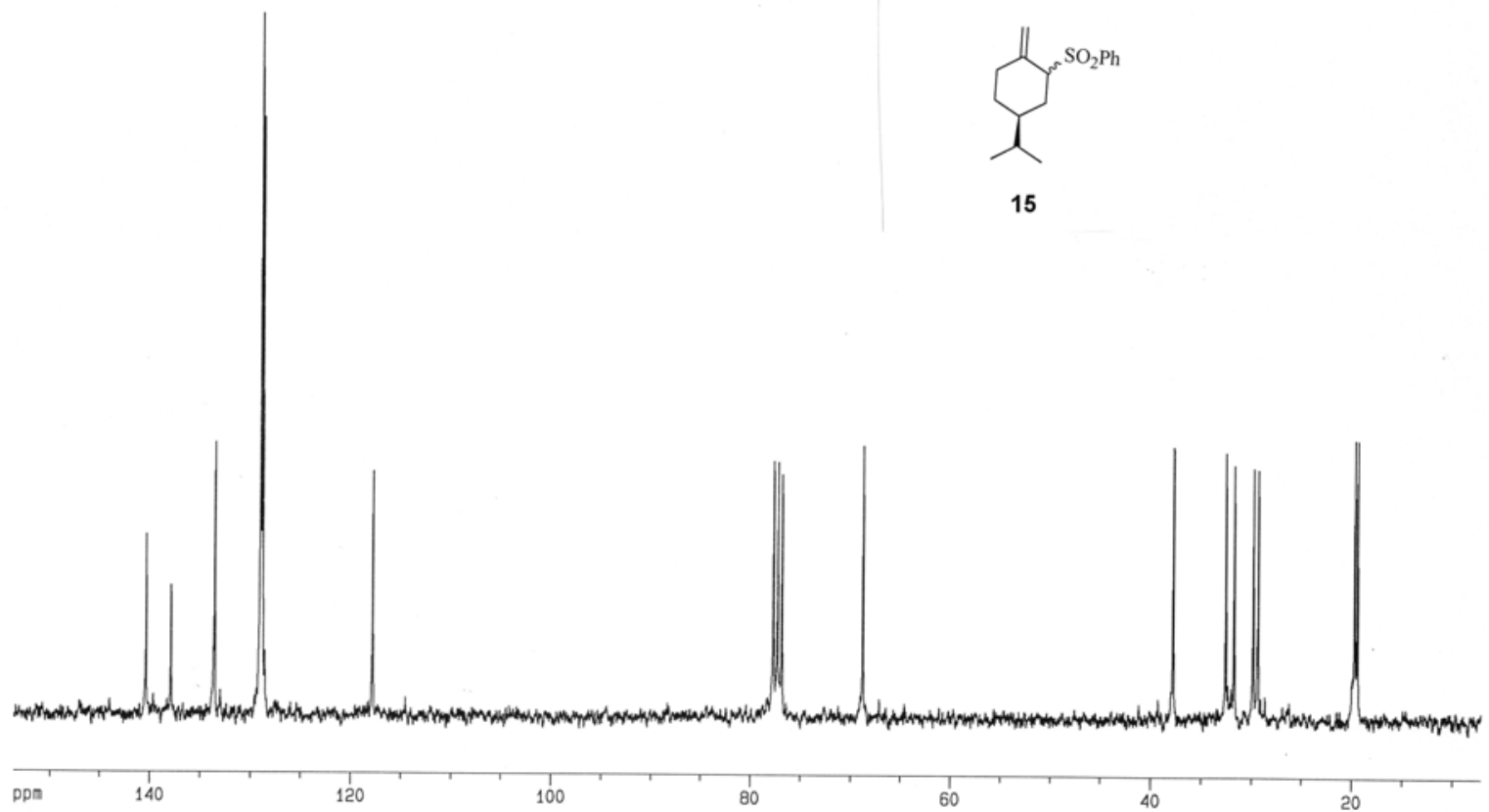




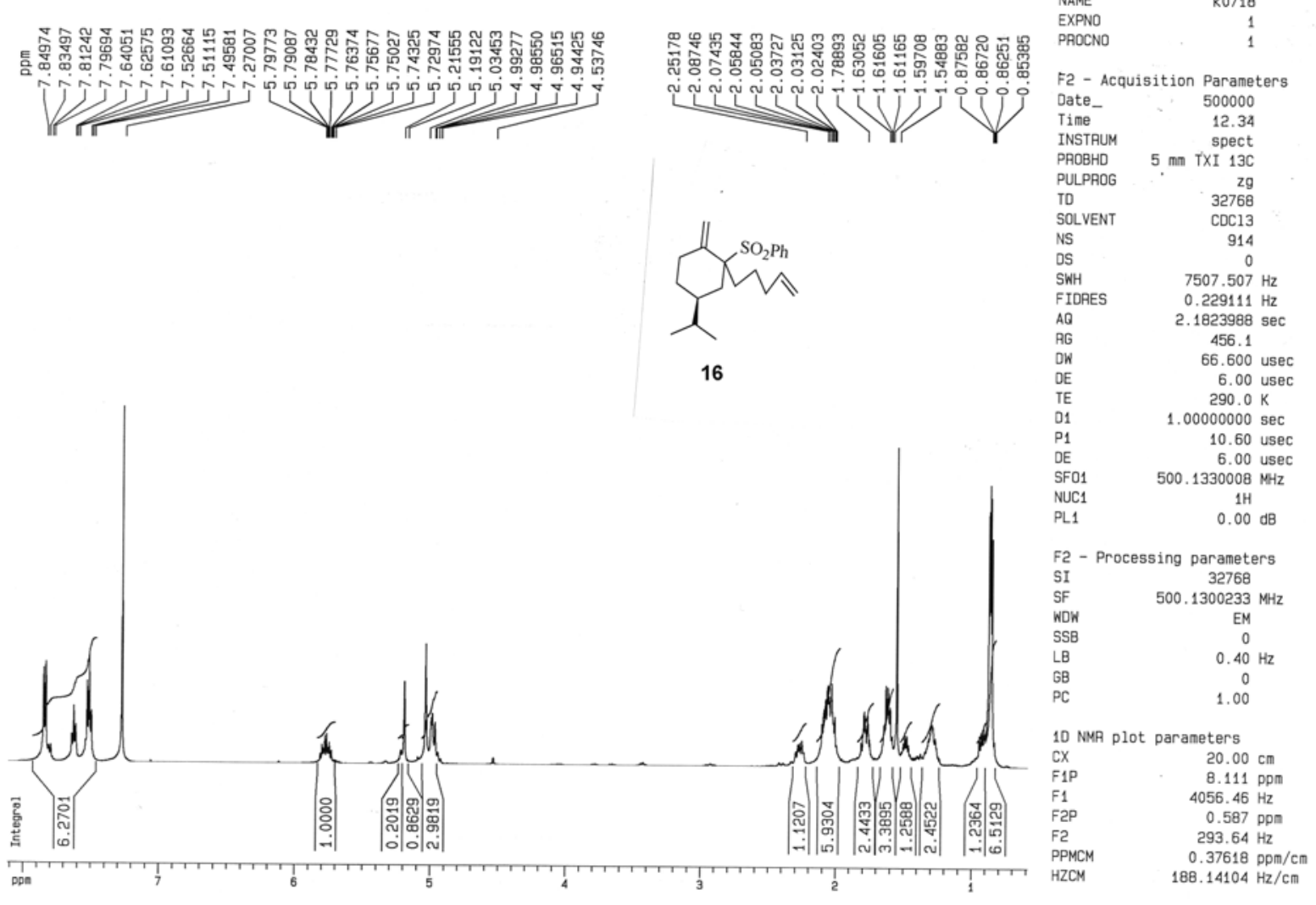




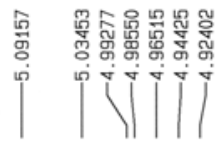

$\begin{array}{lr}\text { Current Data Parameters } \\ \text { NAME } & \text { k0718 } \\ \text { EXPNO } & 1 \\ \text { PROCNO } & 1\end{array}$

F2 - Acquisition Parameters

Date_ 500000

Tine 12.34

spect

PAOBHD $5 \mathrm{~mm}$ TXI $13 \mathrm{C}$

TD

SOLVENT

OS
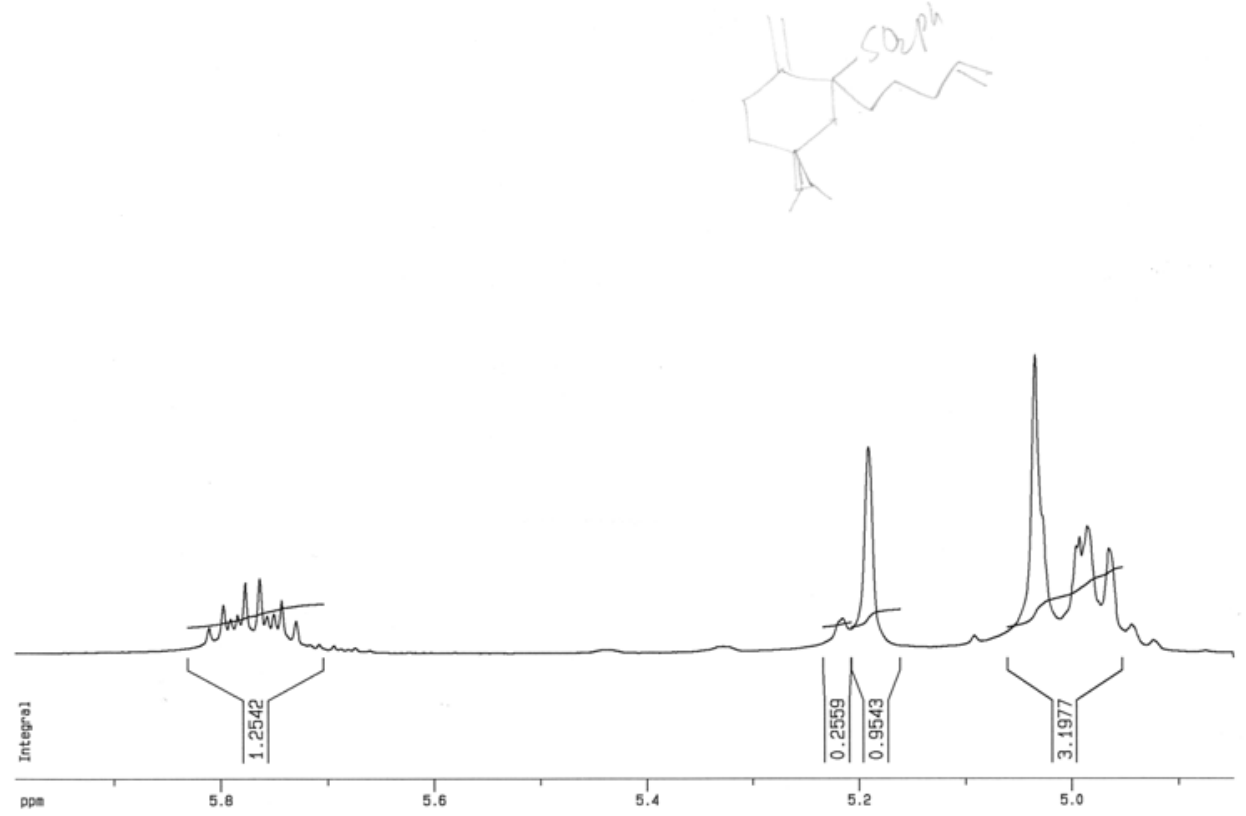

SWH

$\quad 7507.507 \mathrm{~Hz}$

IOAES $0.229111 \mathrm{~Hz}$

$2.1823988 \mathrm{sec}$ 456.1

66.600 usec 6.00 usec $290.0 \mathrm{~K}$ 1. $00000000 \mathrm{sec}$ 10.60 usec $500.1330008 \mathrm{MHz}$

NUC1 $1 \mathrm{H}$

$\begin{array}{ll}\text { NUC1 } & 1 \mathrm{H} \\ \text { PL1 } & 0.00 \mathrm{~dB}\end{array}$

F2 - Processing parameters $\mathrm{SI} \quad 32768$ SF $\quad 500.1300233 \mathrm{MHz}$ $\begin{array}{lc}\text { WDW } & \text { EM } \\ \text { SSB } & 0 \\ \text { B } & 0.40\end{array}$

$\begin{array}{lc}\text { SSB } & 0 \\ \text { LB } & 0.40 \mathrm{~Hz} \\ \text { GB } & 0 \\ \text { PC } & 1.00\end{array}$

10 NMA plot parameters CX $\quad 20.00 \mathrm{~cm}$ $\begin{array}{ll}\text { CX } & 20.00 \mathrm{~cm} \\ \text { F1P } & 5.994 \mathrm{ppm}\end{array}$ F1 $2997.60 \mathrm{~Hz}$ F2P $4.849 \mathrm{ppm}$ F2 $2424.99 \mathrm{~Hz}$ PPMCM $\quad 0.05725 \mathrm{ppm} / \mathrm{cm}$ 


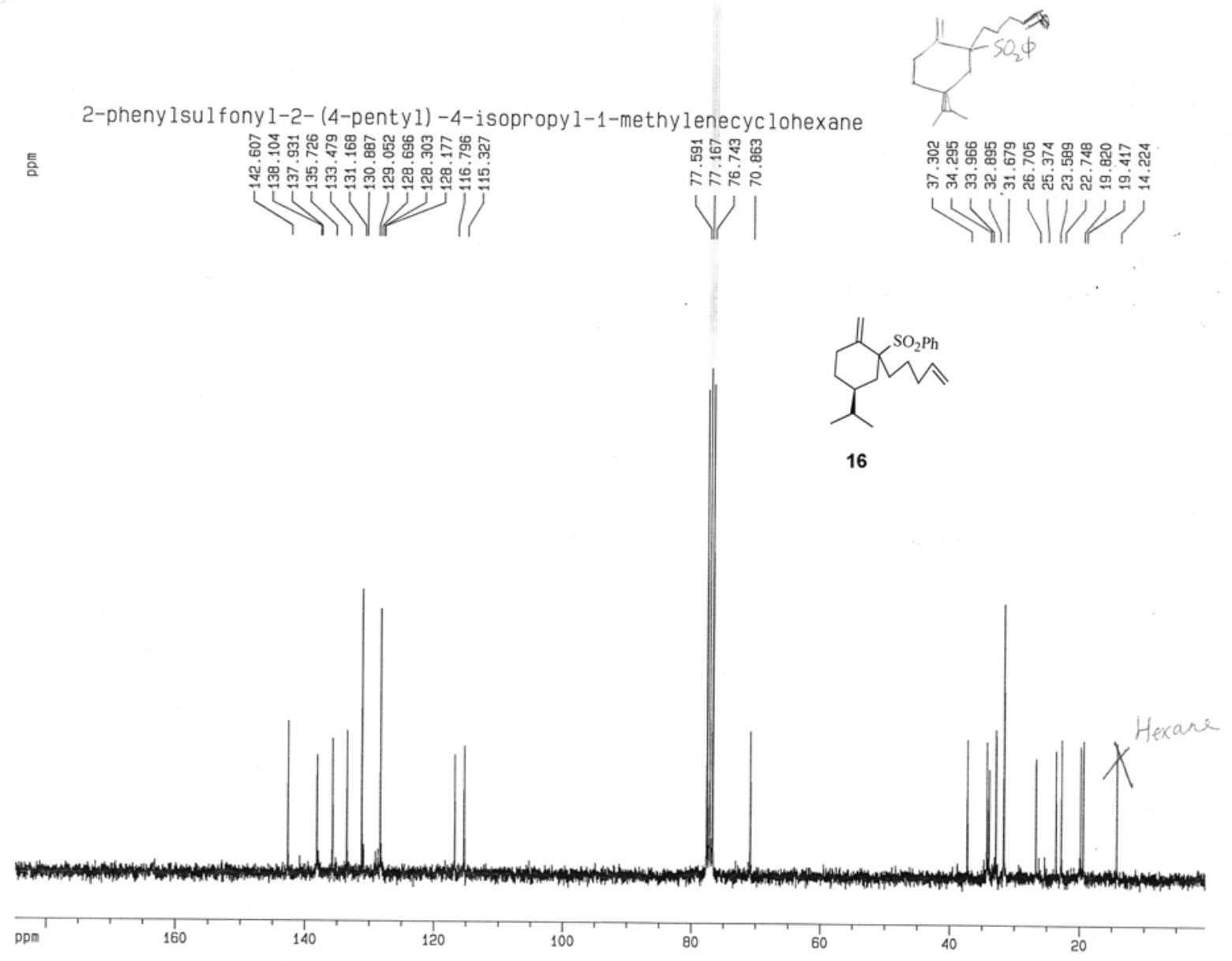




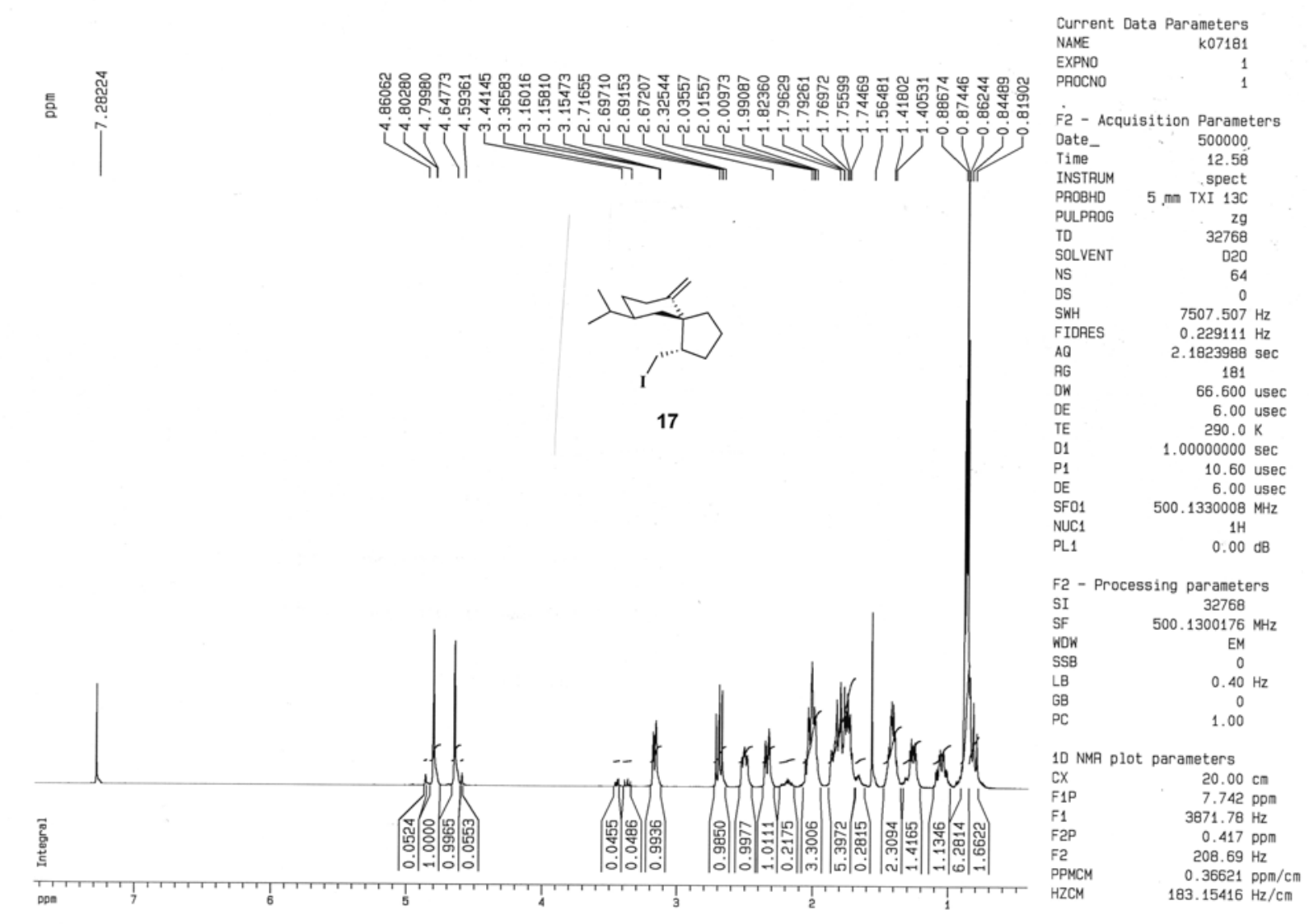




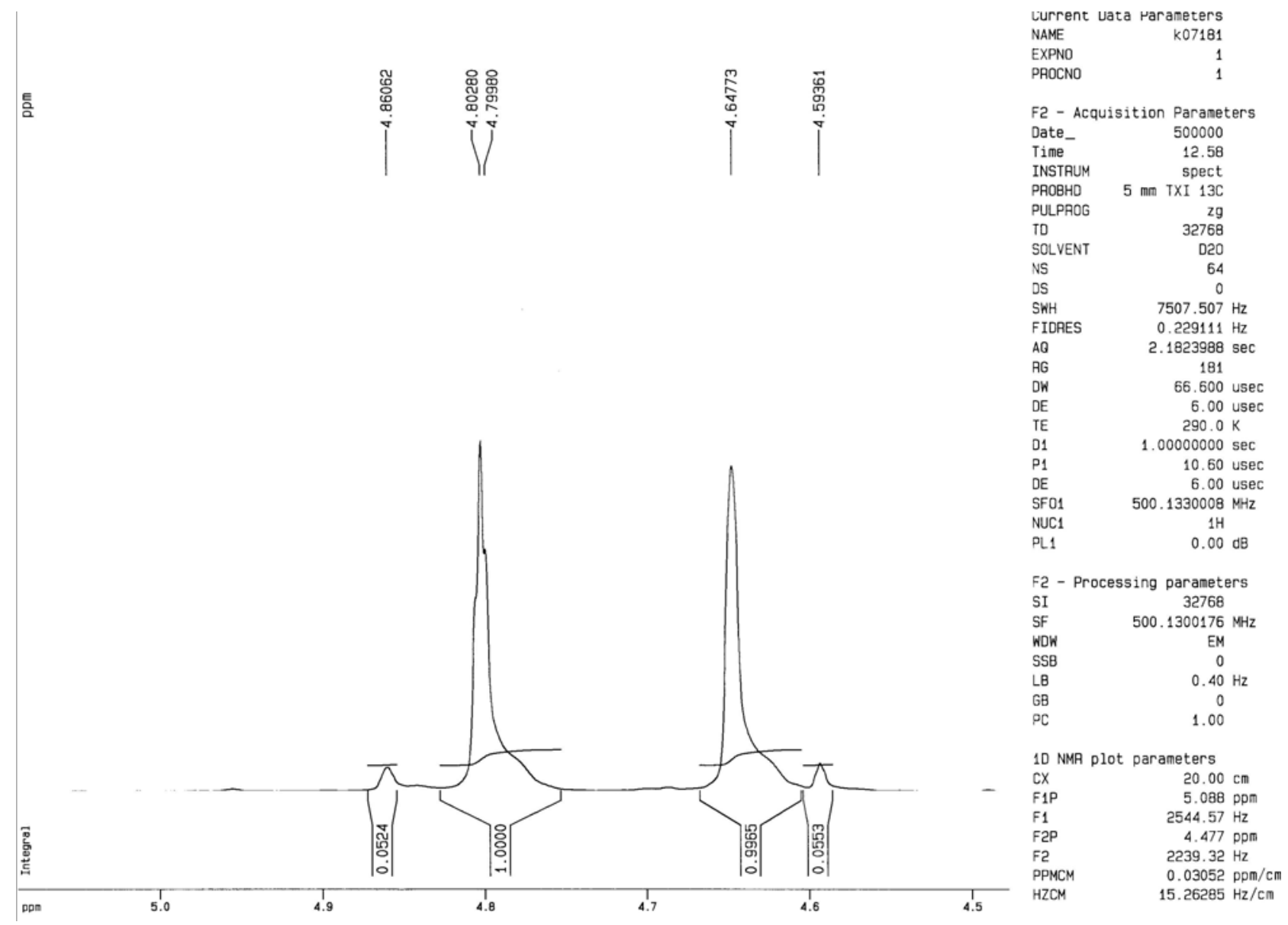




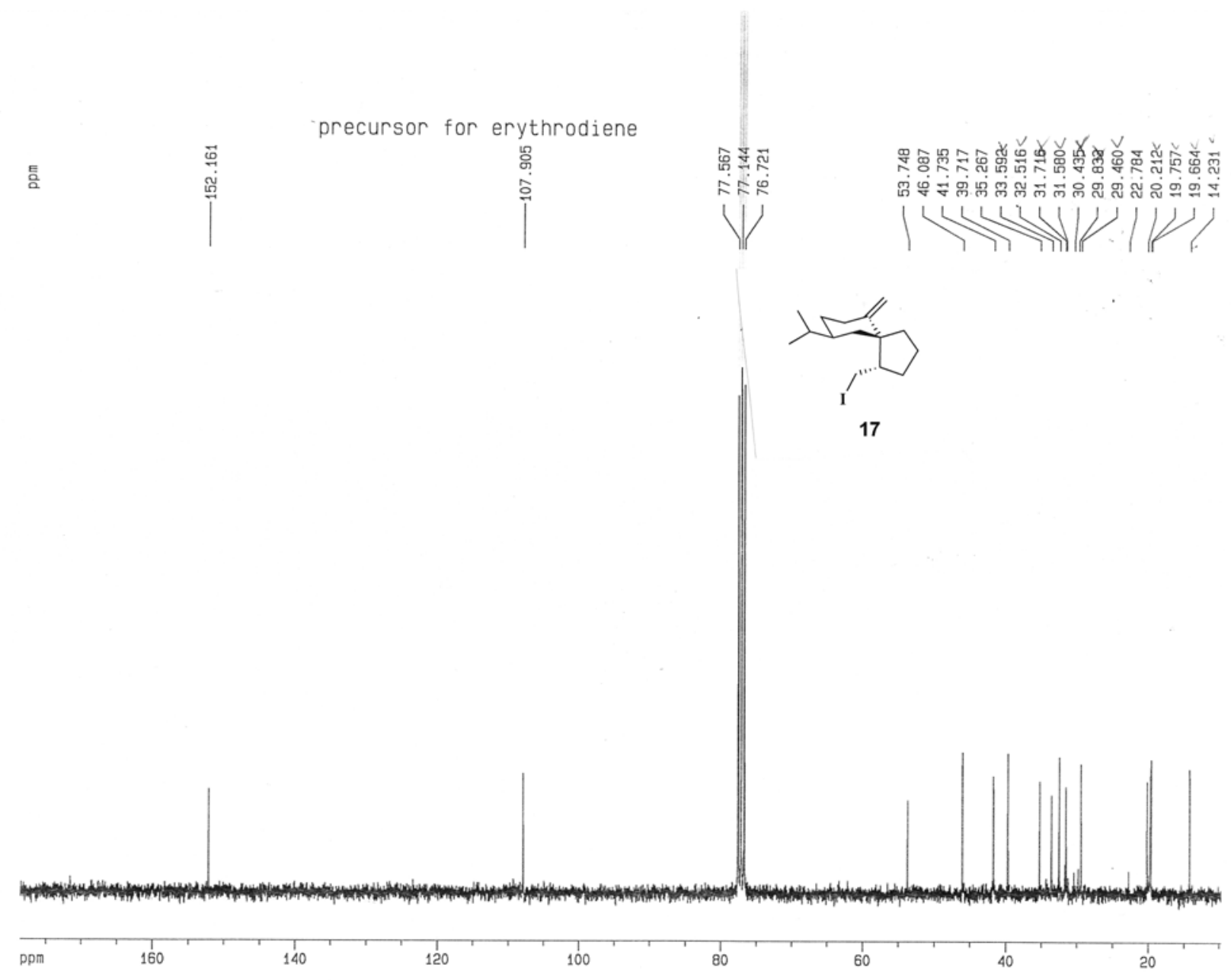




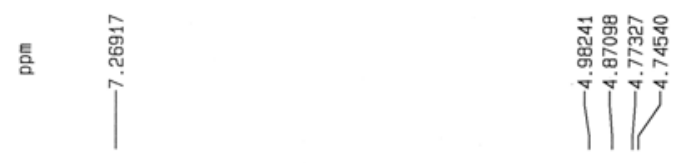

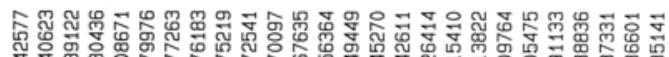
ن

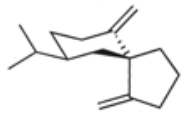

$18(-)$ - Erythrodiene

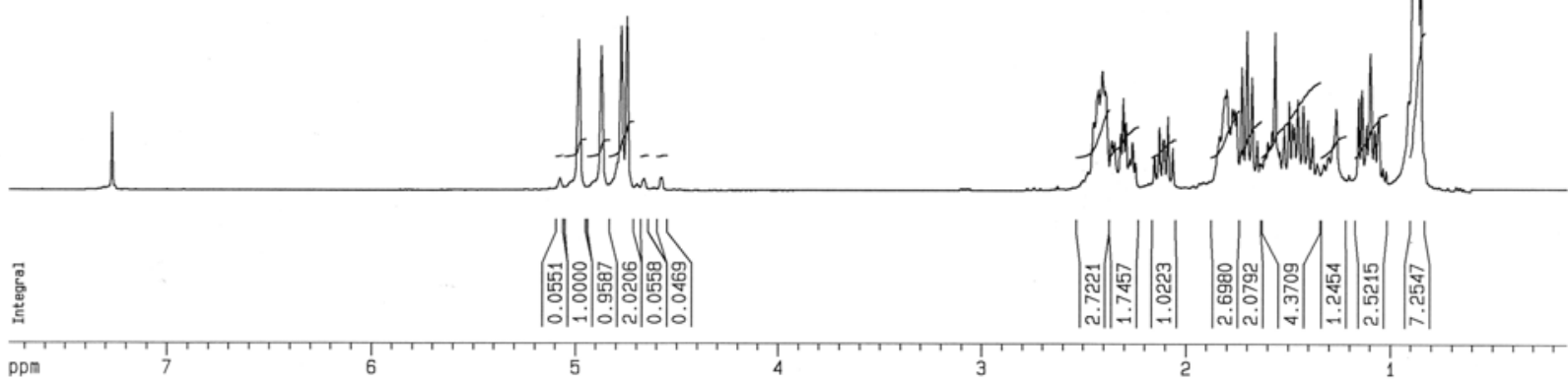


言
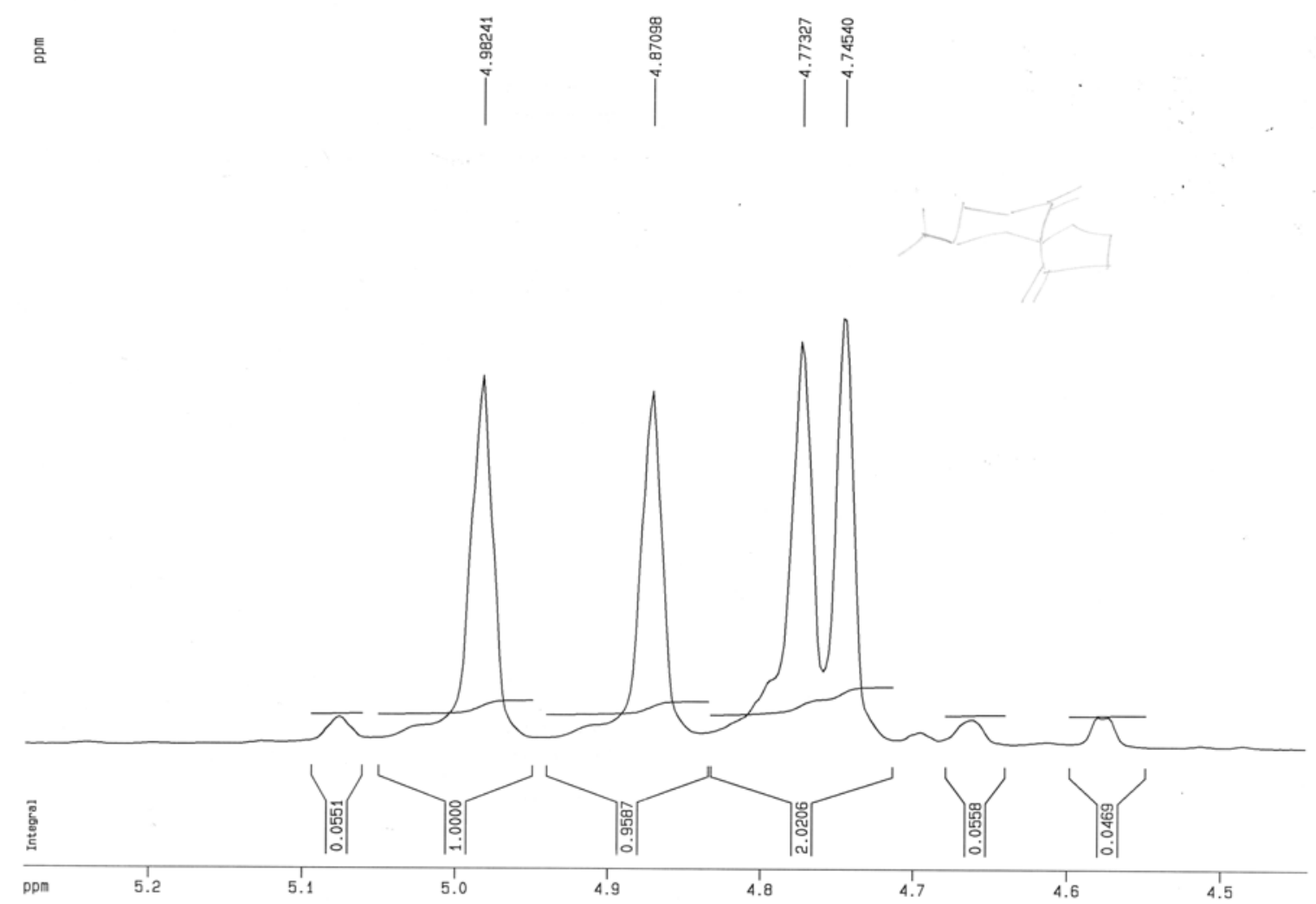
Erythrodiene purified with AgNO3 silica gel

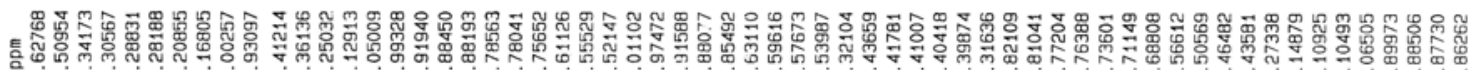

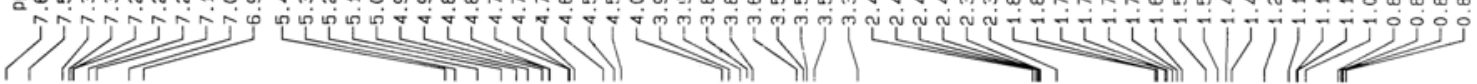

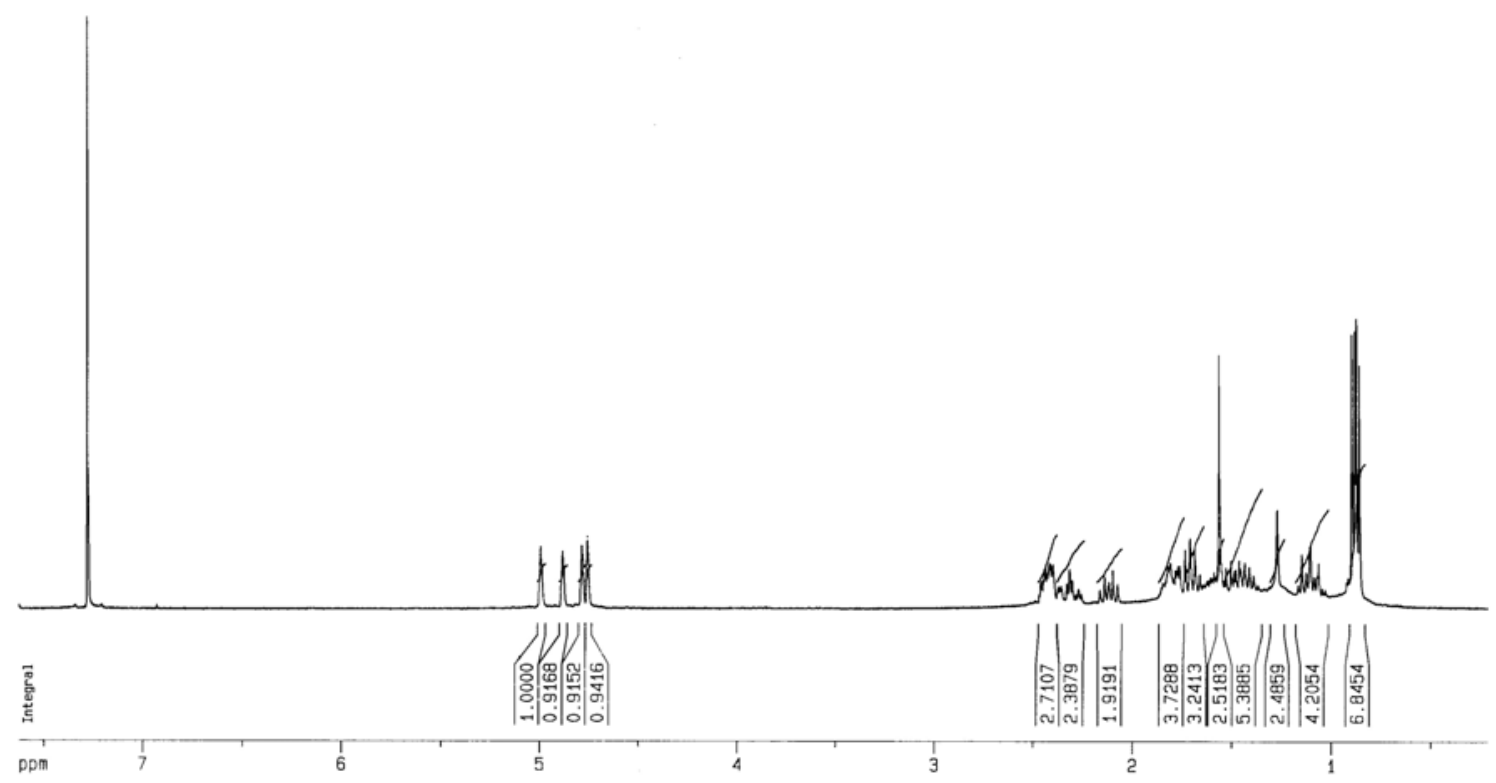




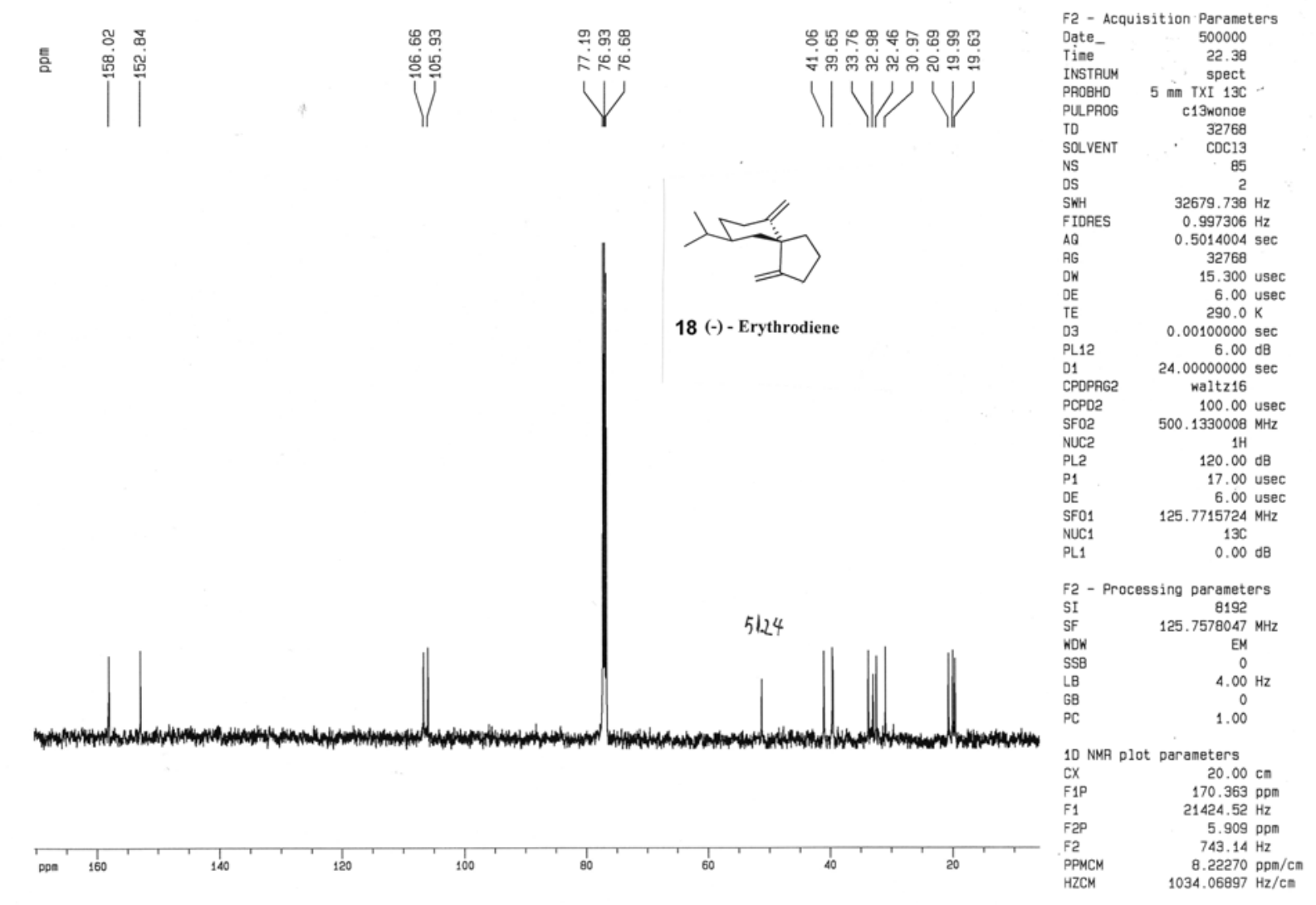



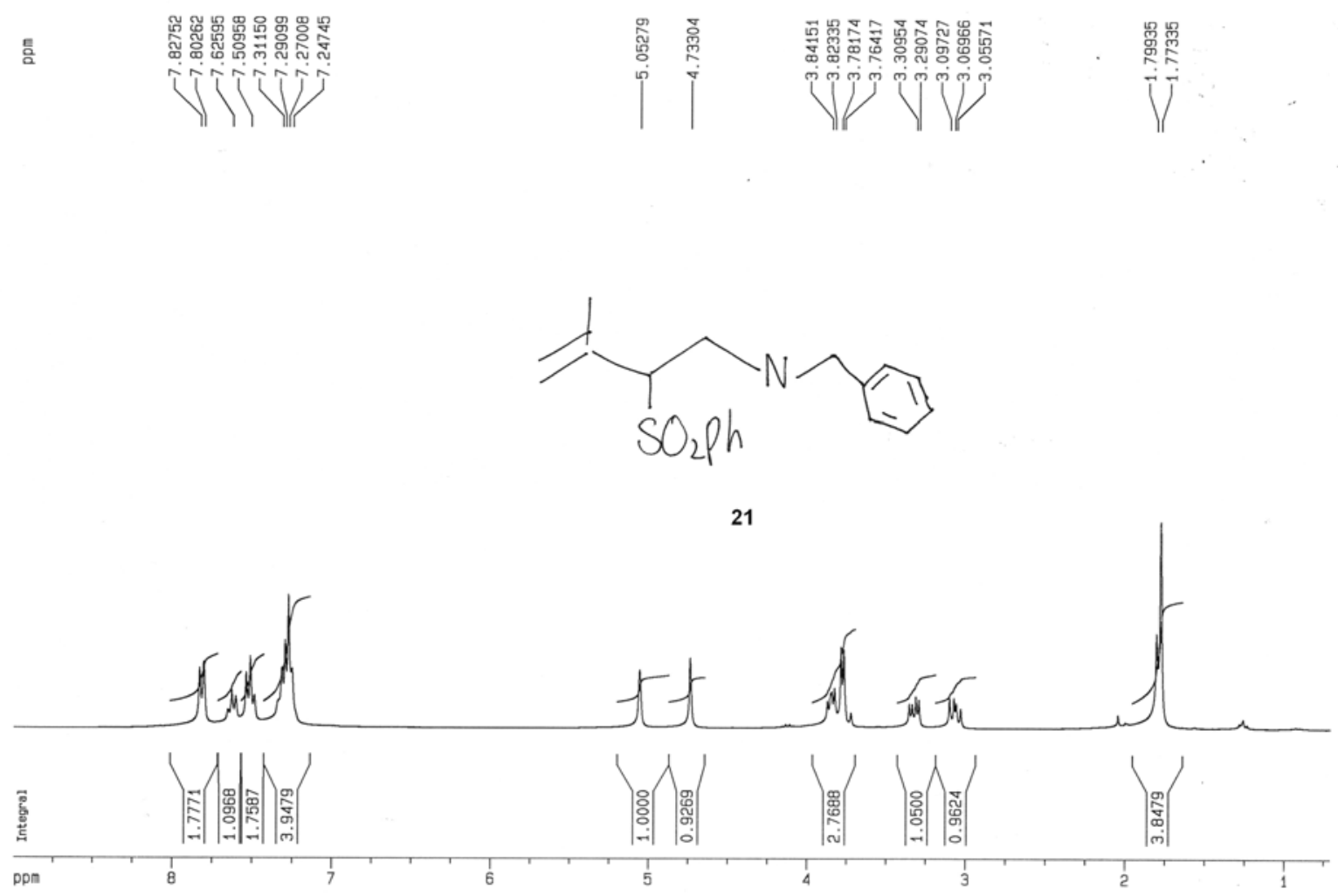

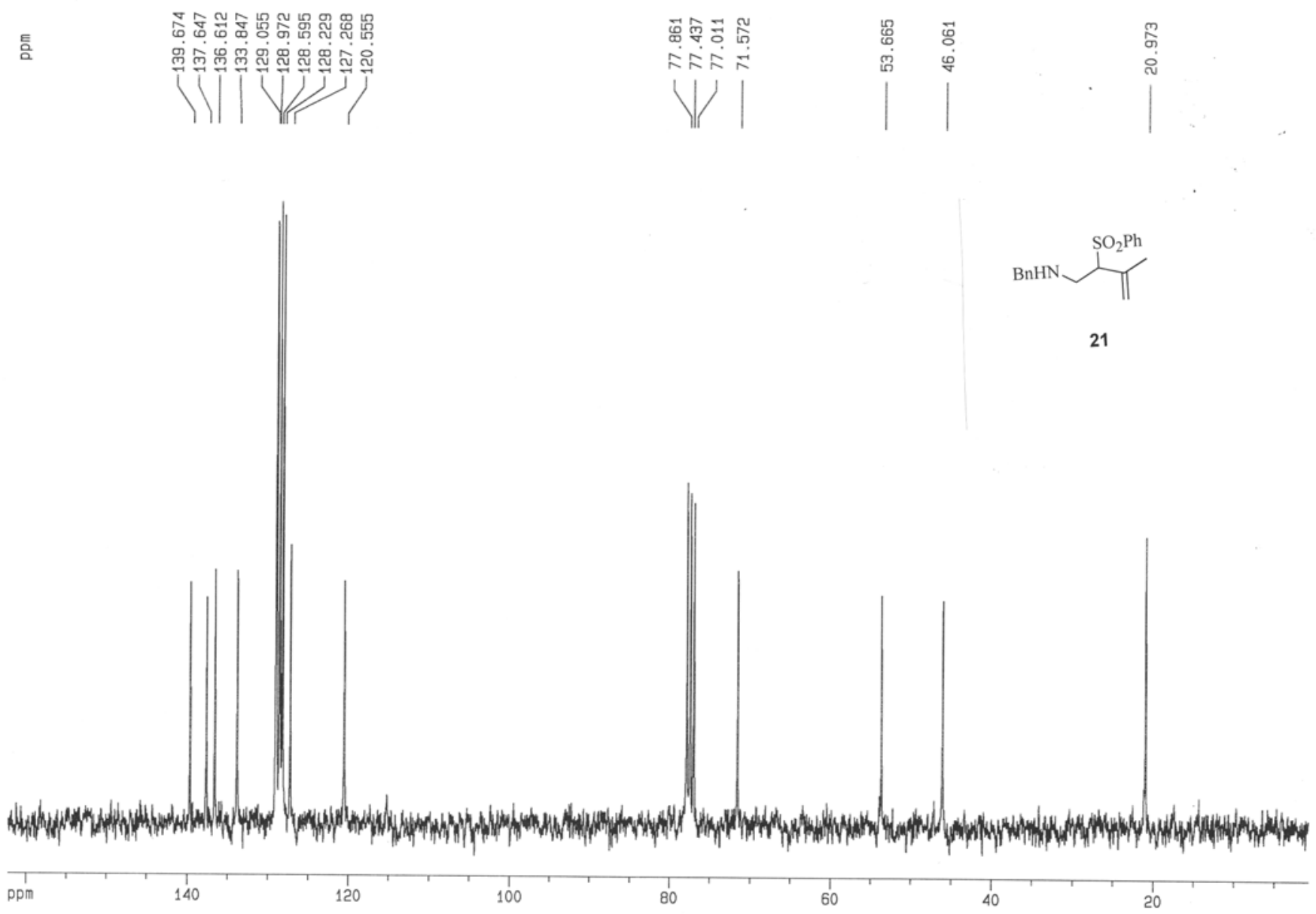

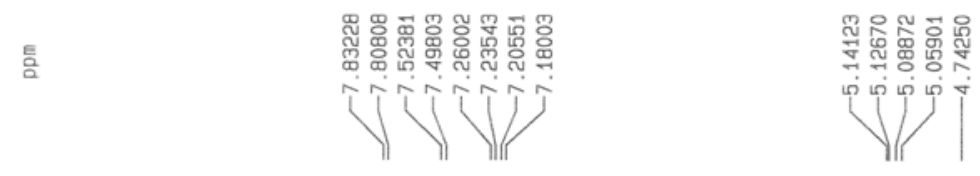

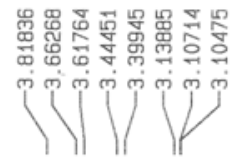
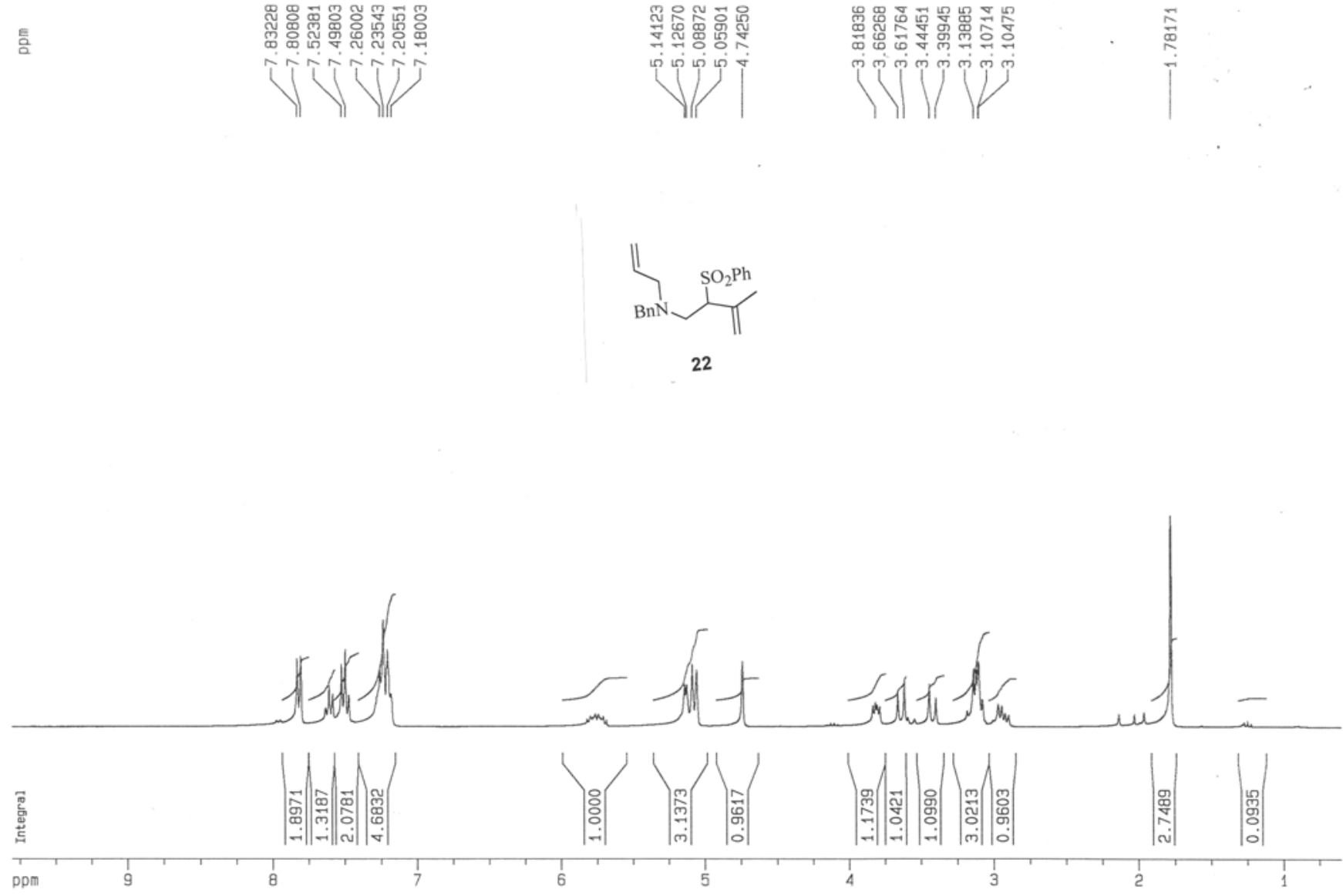

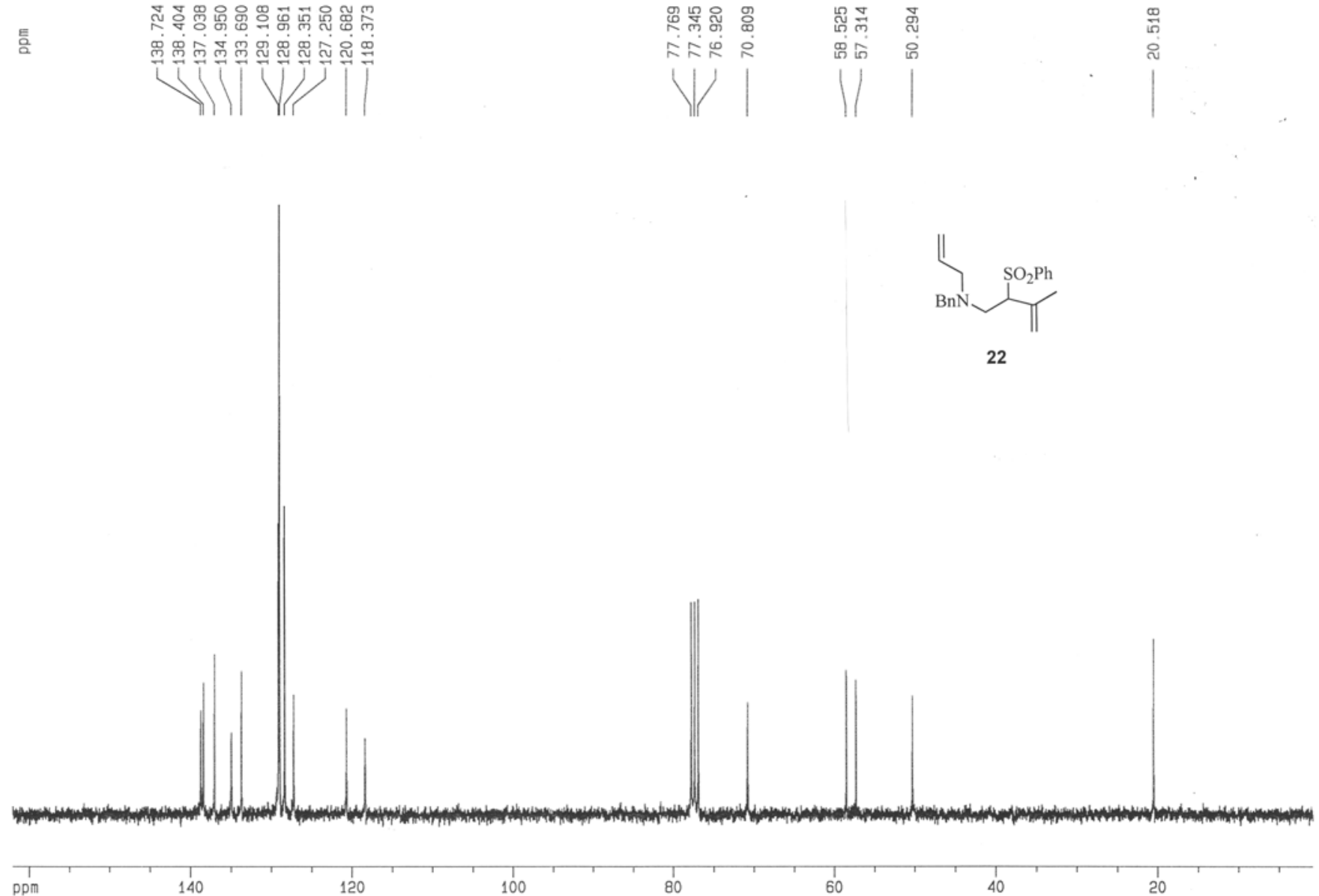

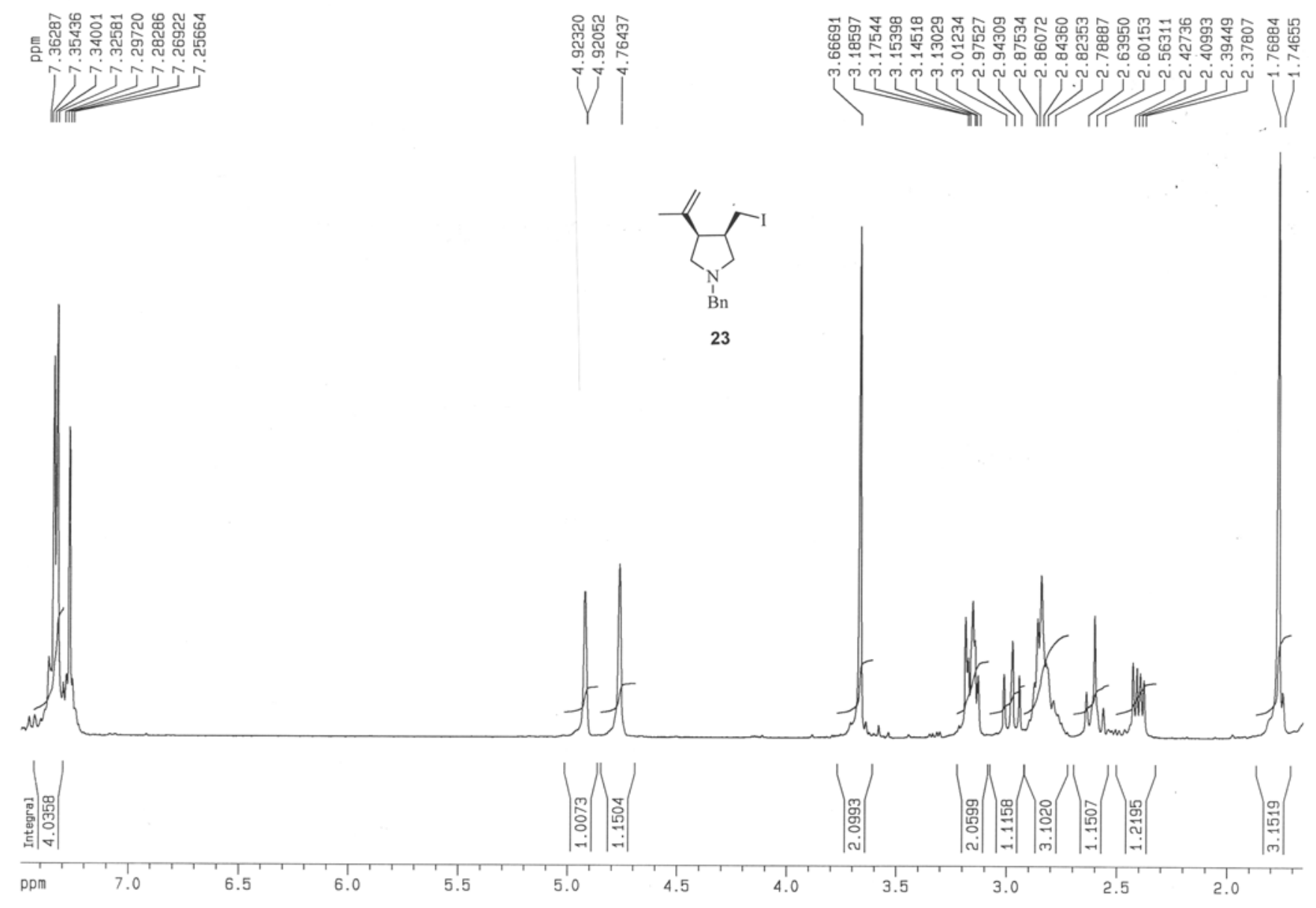


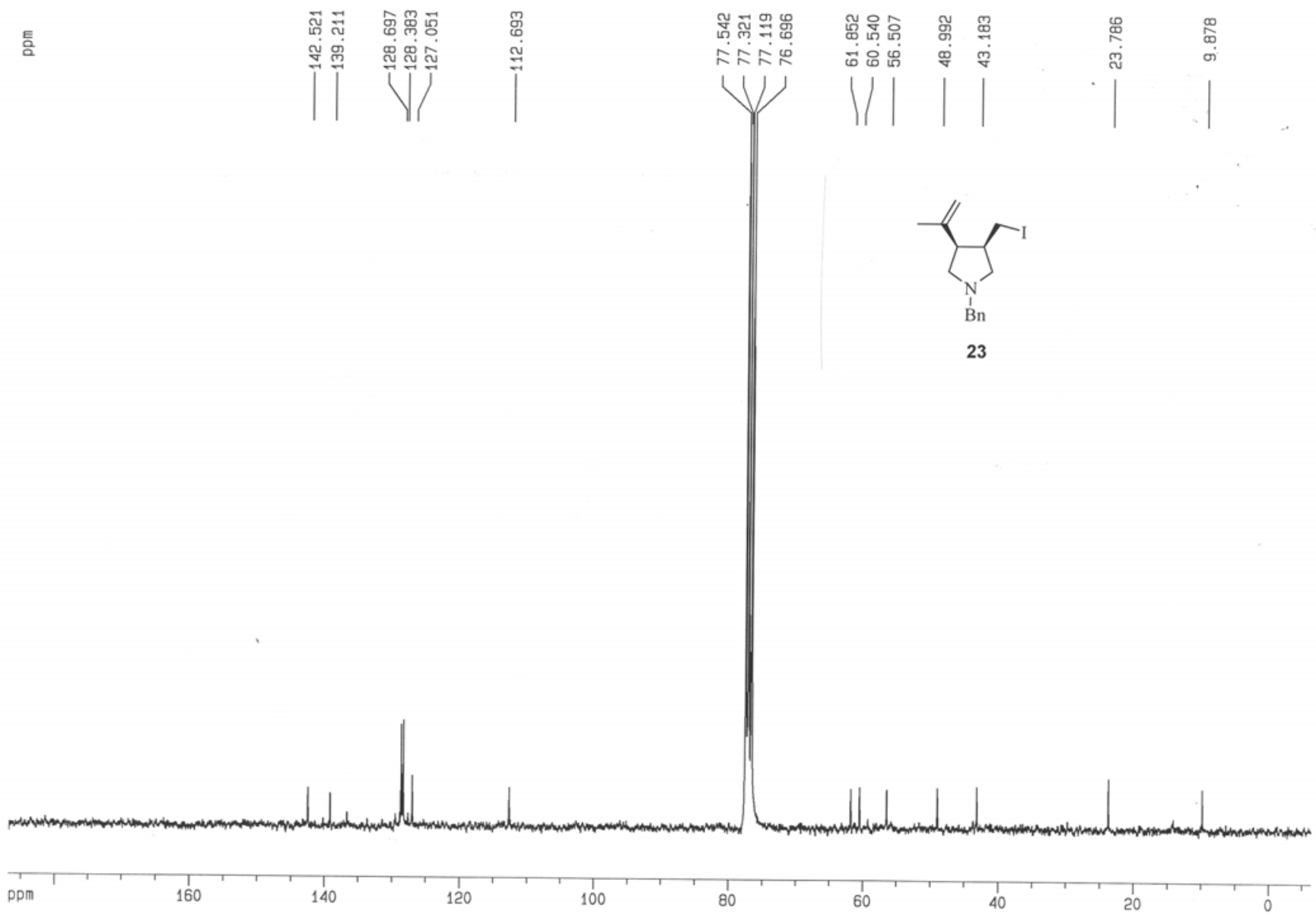

